LBL-663

Journal Preprint

\title{
ENERGY SPECTRA OF FRAGMENTS FROM SILVER AND URANIUM BOMBARDED WITH $5.0 \mathrm{GeV}$ PROTONS
}

Ralph G. Korteling, C.R. Toren, and Earl K. Hyde

August 1972

Prepared for the U.S. Atomic Energy

Commission under Contract W-7405-ENG-48 


\section{ENERGY SPECTRA OF FRAGMENTS FROM SILVER AND URANIUM \\ BOMBARDED WITH $5.0 \mathrm{GeV}$ PROTONS ${ }^{*}$ \\ Ralph G. Korteling and C. R. Toren \\ Department of Chemistry \\ Simon Fraser University \\ Burnaby, British Columbia, Canada \\ and \\ Earl K. Hyde \\ Nuclear Chemistry Division \\ Lawrence Berkeley Laboratory \\ University of California \\ Berkeley, California 94720}

August 1972

\section{ABSTRACT}

In this third paper in a series in which the characteristics of nuclear fragments produced in the interaction of $5 \mathrm{GeV}$ protons with $\mathrm{Ag}$ and $\mathrm{U}$ targets were studied by means of $\mathrm{dE} / \mathrm{dx}-\mathrm{E}$ measurements with semiconductor detector telescopes new information was obtained on the energy spectra of light fragments. One set of measurements on fragments from a Ag target involved the use of a twoelement telescope incorporating a $\Delta \mathrm{E}$ detector as thin as $16 \mu \mathrm{m}$. A new algorithm for processing the $\Delta \mathrm{E}$ and $\mathrm{E}$ data to extract particle identification was developed and the resulting particle spectra showed superior resolution for the elements from $\operatorname{Li}(z=3)$ to $S(z=16)$. Segments of the energy spectra of each of these elements were measured at $20^{\circ}$ and, for many of them, also at $45^{\circ}, 90^{\circ}, 135^{\circ}$ and $160^{\circ}$ to the beam direction. By use of 3-element telescopes and absorbers the high energy part of the energy spectrum for isotopes of $\mathrm{He}, \mathrm{Li}, \mathrm{Be}, \mathrm{B}$, and $\mathrm{C}$ ejected from $\mathrm{Ag}$ and $\mathrm{U}$ targets was measured at $20^{\circ}$. The measurements extended 
beyond $300 \mathrm{MeV}$ for ${ }^{6} \mathrm{Li}$ and ${ }^{7} \mathrm{Li}$ and to $400 \mathrm{MeV}$ for ${ }^{7} \mathrm{Be}$. A distinct highenergy component was found in these cases.

The suitability of nuclear evaporation as a description of the emission of the low-energy fragments was tested with two simple theoretical models, one specifying isotropic fragment emission from a moving nucleus at a fixed nuclear temperature and one specifying isotropic fragment emission from a set of moving nuclei with a Maxwellian distribution of excitation energies and forward momenta. The second could describe rather well all the $90^{\circ}$ data, provided a Coulomb barrier 0.4 that of the classical tangent spheres barrier was used. However, the measured intensity in the forward direction was much higher than predicted. Neither evaporation calculation was able to describe the highest energy part of the spectra, and the conclusion was drawn that these particles must be produced in the initial high-energy cascade. 


\section{INTRODUCTION}

Fragments ejected from complex nuclei during the course of reactions induced by $\mathrm{GeV}$ protons can be studied with silicon semiconductor detectors incorporated in a particle-identifier system. Comprehensive studies of the characteristics of fragments ejected from uranium and silver targets bombarded

by $5.5 \mathrm{GeV}$ protons in the Bevatron have recently been published. ${ }^{1,2}$ The results described in the present paper are supplementary to these previous studies and extend them in two ways.

First, in the case of the silver target study ${ }^{2}$ the data have been extended and improved in the low energy region. For the reasons given in the discussion of the cited papers, ${ }^{1,2}$ it is important to determine the maximum in the energy spectrum of each of the light elements, Li, Be, B, etc. This maximum is determined by the effective Coulomb barrier felt by the fragment, and it is one of the characteristic features of the fragment ejection process that this effective Coulomb barrier is only 40-50 percent as high as the barrier obtained from a tangent spheres calculation. In the previous work the experimental low-energy cut off, which was fixed by the 20 micron thickness of the $\Delta \mathrm{E}$ silicon detector, was so high that it exceeded the maximum in the energy spectrum for all fragments except $\mathrm{He}$ and $\mathrm{Li}$. In the new study, a $16 \mu \Delta \mathrm{E}$ detector was used and, as a consequence, the turnover region of the energy spectrum was defined for $\mathrm{Li}, \mathrm{Be}$, and B. Measurements were made at $20^{\circ}, 45^{\circ}$, $90^{\circ}, 135^{\circ}$, and $160^{\circ}$ to the beam. A special run was made at $20^{\circ}$ to improve the identification of elements up through $\mathrm{Z}=18$ (argon), and a section of the energy spectra of these elements was determined. No attempt was made to remeasure the low energy spectra of fragments from uranium because the turnover regions of the spectra were well defined for $\mathrm{Li}, \mathrm{Be}$, and $\mathrm{B}$ fragments in the original study. 
The second way in which the previous data were extended was in the remeasurement of the ultra-high energy portion of the fragment spectra. One of the interesting features of the previous work was the identification of $\mathrm{Li}$ and $B e$ fragments with energies extending out to $160 \mathrm{MeV}$. In the new study these high-energy fragments were restudied in order to establish beyond question the emission of these ultra-high energy fragments and to measure the energy spectrum out to even higher energies. For this purpose measurements were made on fragments from uranium and silver targets with a three-element semiconductor telescope with a total Si thickness of 1100 to 2200 microns ( 257 to $513 \mathrm{mg} / \mathrm{cm}^{2} \mathrm{Si}$ ). Aluminum absorbers of thickness ranging up to $1.3 \mathrm{~g} / \mathrm{cm}^{2}$ were placed in front of the first detector. In those experiments the energy spectra of isotopes $\mathrm{He}$ through $\mathrm{C}$ were measured at $20^{\circ}$ to the beam. In the case of ${ }^{6} \mathrm{Li}$ and ${ }^{7} \mathrm{Be}$ the energy spectra were measured out to $340 \mathrm{MeV}$ and $420 \mathrm{MeV}$, respectively.

Section II describes the experimental techniques for data taking and reduction. Section III presents the results of the restudy of the low energy portion of the energy spectra as well as a discussion of these results. The high energy data are presented and discussed in Section IV. Section V summarizes the main conclusions of the paper. Appendix A is devoted to a new method of determining particle identity from $\Delta E$ and $E$ detector signals. Appendices $B$ and $\mathrm{C}$ cover mathematical details on two evaporation formulas used in the analysis of the energy data. 


\section{EXPERIMENTAL}

Inasmuch as the experimental techniques were nearly the same as those described in Ref. 1, they are presented here in shortened form except for details of the detectors or for changes from the earlier methods.

\section{A. Bombardment Arrangement at Bevatron}

Thin targets of metallic Ag or $U$ were placed in the center of a 36 inch diameter target chamber located in one of the external beam lines of the Bevatron. This chamber could be isolated from the beam line by gate valves in the upstream and downstream ports. A mechanical pump system was provided for evacuation of the chamber to the 5-10 micron level, which was the normal pressure for the beam line. It was necessary to achieve a better vacuum for operation of the $2000 \mu \mathrm{E}$ detectors used in the second part of the study because bias voltages up to 1500 volts were needed. This better vacuum was obtained by installing 0.003 inch aluminum windows beyond the inlet and outlet valves and by pumping the chamber volume with a cryogenic pump. With this arrangement a much lower vacuum was maintained in the chamber and no problems with sparking or voltage breakdown occurred.

The silver target for the low energy experiments was prepared by volatilization of metallic silver to make a uniform foil of $1.1 \mathrm{mg} / \mathrm{cm}^{2}$. This was glued across a hole in a 0.00025 inch mylar sheet which in turn was stretched across a $3.5 \times 6$ inch hole in an aluminum frame. The target was positioned at $55^{\circ}$ to the beam for fragment measurements made at $20^{\circ}, 45^{\circ}$, and $90^{\circ}$. It was placed at $135^{\circ}$ for measurements at $135^{\circ}$ and $160^{\circ}$. For measurements of fragments of higher energy silver foil targets with uniform thicknesses of $7 \mathrm{mg} / \mathrm{cm}^{2}$ and $26 \mathrm{mg} / \mathrm{cm}^{2}$ and a uranium foil target with a thickness of $27.5 \mathrm{mg} / \mathrm{cm}^{2}$ were used in similar target frames. 
The external beam of $5.0 \mathrm{GeV}$ energy was delivered in pulses of 800 to $1000 \mathrm{msec}$ duration repeated every 6 seconds and containing about $8 \times 10^{11}$ protons per pulse. The beam cross section at right angles to the beam integrated over a period of hours was determined by radioautographs to be roughly $1 / 2$ inch vertically by $1-1 / 2$ inch horizontally. The cross section of the target foils was somewhat larger.

The telescope of silicon semiconductor detectors was mounted on an aluminum block which in turn was clamped to a movable arm whose pivot point was at the chamber center.

\section{B. Detector and Electronic Details for Measurement of Low Energy Fragments}

The telescope had two elements: a $16 \mu \mathrm{m}$ thick surface-barrier detector used as the $\Delta E$ detector and a $194 \mu \mathrm{m}$ thick planar detector of phosphorus-diffused silicon used as the $\mathrm{E}$ detector. Behind these there was also a $381 \mu \mathrm{m}$ thick planar detector of phosphorus diffused silicon used as the rejection detector to eliminate the recording of high-energy fragments which were not stopped in the $\mathrm{E}$ detector. Collimators with $5 \times 7 \mathrm{~mm}$ openings cut into 0.125 inch copper were placed in front of the $\Delta \mathrm{E}$ and the $\mathrm{E}$ detector. The distance between the two collimators was $2.8 \mathrm{~cm}$. (In the 3-element telescope described in Sec. II.C. the collimators were positioned in front of the first and third detectors and were separated by $2.1 \mathrm{~cm}$.)

The electronic circuitry was identical with that illustrated in Fig. I of Ref. 2. In brief, this circuitry accomplished the following purposes. Pulses from the $\Delta E$ and $E$ detectors were not accepted unless they were received within a $50 \mathrm{nsec}$ resolving time. If a pulse was simultaneously recorded in the 
$\mathrm{E}_{\text {reject }}$ detector the event was discarded. A pile up rejector circuit inspected the preamplifier output and excluded any events in which a second pulse was received within an inspection period of $600 \mathrm{nsec}$. The output pulses from the main amplifiers were passed through single channel analyzers which accepted all pulses above $1.5 \mathrm{MeV}$ in the $\Delta \mathrm{E}$ detector and $2 \mathrm{MeV}$ in the $\mathrm{E}$ detector. Pulses surviving these tests were accepted for digitization by an analog-to-digital converter interfaced to a PDP-8 Computer and were written on magnetic tape in blocks of 128 events. These records were later processed at a larger computer as discussed below. The surviving $\Delta \mathrm{E}$ and $\mathrm{E}$ pulses were also transmitted to a Goulding-Landis particle identifier system ${ }^{3}$ for analog development of a particle identifier signal which was sent to a pulse height analyzer for recording and display. The on-line display of a histogram of the analog particle identifier signal was used for monitoring and trouble-shooting purposes during the datataking portion of the experiment, but all data presented in this paper resulted from off-line computer calculations with the original digitized pairs of $\Delta \mathrm{E}$ and $\mathrm{E}$ pulse heights, as discussed in Appendix A and Sec. II.E. As discussed in Ref. 1 precision pulsers were used for the energy calibration.

\section{Detector and Electronic Details for Measurement of High Energy Fragments}

The three different 3-element telescopes used in this part of the experiment are listed in Table $I$. The $\Delta \mathrm{E}$ and $\mathrm{E}_{r e j}$ detectors were all manufactured of phosphorus diffused silicon. The $1000 \mu$ and $2000 \mu \mathrm{E}$ detectors were silicon surfacebarrier detectors. The $1500 \mu \mathrm{E}$ detector was made of lithium drifted silicon. It was our experience that lithium drifted detectors could be used only for a short time ( 12 to 24 hours) in close proximity to the beam before radiation damage effects, probably involving precipitation of lithium, caused deterioration 
of the detector signals, particularly the timing characteristics of the signal. Data whose quality was seriously affected by these radiation damage effects were discarded. The surface barrier detectors also suffered radiation damage in this hostile environment, manifested mainly as a steady increase in leakage currents which rose steadily from a few $\mu$ amps up to more than 50 uamps over several days of exposure. However, the energy and time characteristics of the pulses were not seriously affected and the quality of the data remained good. The electronic circuitry associated with the 3-element telescope was identical with that displayed in Fig. 1 of Ref. 1 and fully discussed in that paper. Coincidence requirements, pile up rejection, and pulse height discrimination were in principle the same as those briefly mentioned in $B$ above. One change in the use of the pile up rejector circuitry was the elimination of inspection of the $\mathrm{E}$ detector pulse because the long collection times for ionization pulses in these thick detectors produced preamplifier output pulses too slowly rising for the use in the pile up rejector. Also the role of the analog particle-identifier system was more important for these telescopes incorporating two $\Delta \mathrm{E}$ detectors. By analog circuitry a particle identify pulse was generated from the $\Delta E_{2}$ and the $E=\Delta E_{1}+E$ pulses and compared with a second identity pulse generated from the $\Delta \mathrm{E}_{1}$ and $\mathrm{E}$ inputs. If the two identifications did not agree within a preset amount (in most cases $15 \%)$ the event was rejected and the $\Delta E$ and $E$ signals were not sent to the computer for permanent recording. Not many events were rejected by this requirement $(<10 \%)$ but the quality of isotopic resolution was improved. The final particle identification was made off-line by use of the $\Delta \mathrm{E}_{2}$ and $\Delta \mathrm{E}_{1}$ sum as the $\Delta \mathrm{E}$ value in a calculation performed according to the method described in Appendix A. 
The total thickness of silicon in the detector stacks listed in Table I is not sufficient for exploring the range of fragment energies of interest in this study. We could have increased this thickness by using thicker $\Delta \mathrm{E}$ detectors and thicker and more numerous $\mathrm{E}$ detectors, but this would have introduced greater complexity in the electronic circuitry as well as special difficulties with the coincidence timing, and with the corrections for the dead layers on the extra detectors. We decided instead to extend the measured energy range by placing various thickness of absorber directly in front of the first detector in the telescope. Four absorbers were machined of aluminum stock with thickness $0.0052,0.063,0.1004$, and 0.189 inches $(35.7,432,690$ and $1295 \mathrm{mg} / \mathrm{cm}^{2}$, respectively).

These thicknesses were chosen after detailed calculation of the expected energy losses for different particles in the absorber and in the detectors. A computer program called LAZY written by Dr. Creve Maples ${ }^{4}$ was modified for this purpose. In our version of the program the range relationships of P. G. Steward ${ }^{5}$ were incorporated. These calculations helped greatly in the choice of absorbers and detector thicknesses, energy thresholds for the detectors, and other parameters in the experimental setup.

Consideration was given to the possibility that the thick aluminum absorbers would cause multiple scattering of the fragments sufficiently great to affect the intensity of measured fragments. However, the fact that the absorber was mounted directly in front of the collimator attached to the front of the first $\Delta \mathrm{E}$ detector greatly reduces the change of loss by scattering and even in the worst case, which is the scattering of $\mathrm{Li}$ by the $189 \mathrm{mil}$ absorber, a calculation showed that the effect is negligibly small (< $1 \%)$. 
The possibility of an appreciable reaction of the fragments with the aluminum absorber was also considered. For a reaction cross section assumed equal to the geometrical cross section for fragments above the Coulomb barrier, it was estimated that 5 percent or less of the fragments reacted during passage through the thickest absorber. This is within the experimental error for measurement of fragment intensity .

Table I summarizes the specific combinations of telescope and absorbers used to obtain the data discussed in Sec. IV.

The integrated beam through the target was measured with a monitor telescope identical to that described in Refs. I and 2. The factors needed to convert the ratio of counts in the fragment and monitor telescopes into an absolute reaction cross section were obtained from these earlier studies. The ultimate standard of intensity was the ${ }^{7}$ Be cross section determined by radiochemistry as discussed in the appendix of Ref. 1. In the runs with the $\mathrm{Ag}$ target and the $16 \mu \mathrm{m} \Delta \mathrm{E}$ detector the energy windows in the monitor telescope were incorrectly set and the normalization was done in a more indirect manner; the ${ }^{7} \mathrm{Be}$ spectrum at $90^{\circ}$ was normalized to the corresponding spectrum of Ref. 2 and the resulting normalization factor was applied to all data from these runs.

\section{Reduction of $\Delta \mathrm{E}$ and $\mathrm{E}$ Data}

Generation of Particle Spectra. The magnetic tapes with the event-byevent record of $\Delta \mathrm{E}$ and $\mathrm{E}$ signal information were processed by the method described in Appendix A. A computer program called PICAL was used. Generation of Energy Spectra. The output tape of the PICAL program, containing the summed data on PI vs $E_{T}$ in the form of a $1024 \times 1024$ matrix, was used as input to another program (SAVEX) which performed the following functions. 
The data were sorted by PI windows, which defined specific particles, and the energy data for these selected particles were isolated. At this point the energy scale was an arbitrary one set by the gains in the amplifiers and by the analog-to-digital converter. The absolute energy scale for energy deposited in the detectors was computed by use of data taken with a calibrated pulser. The fragment energies were then corrected as in Ref. 1 for the losses incurred in escaping from the target and in passing through the thick aluminum absorbers. This correction was based on a power-law, range-energy relationship, $R=a \varepsilon^{b}$ with sets of parameters ( $a$ and $b$ ) fitted to different energy ranges as described by Bichsel and Tschalaer. 6 This correction was a major one in the case of the thick absorber experiments and it was complicated by the fact that in the process of working backward from the energy deposited in the detector to the energy incident on the absorber it was necessary to use several sets of power law parameters to cover the broad energy range. Cross checks were made between the results of this procedure and the entirely independent method employed in the above-mentioned LAZY program ${ }^{4}$ which depends on the range-energy method of Stewart. 5 Agreement within a few $\mathrm{MeV}$ was found. In the case of element spectra the range-energy relationship of the most prominent isotope was used. The ambiguity in the correction caused by this procedure was negligible for all low-energy results for lighter elements discussed in Sec. III. The uncertainty in the correction was several $\mathrm{MeV}$ in the case of unseparated isotopes, such as unresolved ${ }^{9} \mathrm{Be}+{ }^{10} \mathrm{Be}$, in the thick absorber data discussed in Sec. IV. The intensity scale was converted to an absolute cross section scale by multiplying the monitor telescope count by a calibration constant taken from the previous work. ${ }^{1,2}$ The end result of the SAVEX program was a set of printed tables of double differential cross section data and plotted curves of the same data for selected fragments. 


\section{RESULTS AND DISCUSSION OF LOW ENERGY DATA}

\section{A. Particle Spectra}

Figure 1 shows the particle spectrum derived from data taken at $20^{\circ}$ to the beam with telescope 1 of Table I and analyzed according to the procedure discussed in Appendix A. This run represents a data-taking period of $\sim 50$ hours, the longest of any of the experiments. This length of time enabled us to obtain significant statistical accuracy for the elements up through sulfur (element 16). A comparison of this figure with Fig. 3 of Ref. 2 shows that better statistical accuracy and better elemental resolution was achieved in the present study.

Beryllium-7 is well separated from ${ }^{9,10}$ Be but otherwise no isotopic resolution is apparent; this is a consequence of the thinness of the $\Delta \mathrm{E}$ detector.

Spectra similar to Fig. 1 were generated from data taken at $45^{\circ}, 90^{\circ}$, $135^{\circ}$, and $160^{\circ}$ with good resolution of the elements Li through Na. Figure 2 shows the $90^{\circ}$ results which are typical of intensity levels for the low energy measurements at these 5 angles (about 12 hours per run).

\section{B. Energy Spectra}

Figure 3 shows the energy data for the element lithium at 5 angles. Data points from the present measurements are sums over $2 \mathrm{MeV}$ energy intervals. A part of the data from Ref. 2 was included in Fig. 3 to indicate the trend of the energy spectra between 60 and $160 \mathrm{MeV}$ at 5 angles. The dashed curves in the figure represent an attempt to fit the shape of the spectra up to $35 \mathrm{MeV}$ to the predictions of a very simple evaporation expression taken from Refs. I and 2, which assumes a uniform nuclear temperature for the ensemble of evaporating nuclei. A brief statement of this evaporation expression is given in Appendix $B$. As in Ref. 2 families of curves were computed for a variety of parameter choices and compared with the experimental data to arrive at the set of "best" 
values listed in Table II. Values from Ref. 2 are also listed there. The differences in the two sets result from the fact that those from the present work were chosen for the best fit of the data up to $35 \mathrm{MeV}$ whereas those from Ref. 2 were chosen for the best fit up to somewhat higher energy

A similar presentation of energy spectra and theoretical curves for $7 \mathrm{Be}, 9,10_{\mathrm{Be}}$, boron, and carbon is given in Figs. 4 through 7 . In all cases the parameters used for the calculated (dashed) curves are those given in Table II. Also, in all cases the data at the five angles are shown displaced for clarity by factors of 10 on the intensity scale. Figures 8 through 10 present data on $\mathrm{N}, \mathrm{Ne}$, and $\mathrm{Na}$. Figure 11 is a composite of $20^{\circ}$ data for the elements $\mathrm{B}$ through S.

The comparison of the data of Figs. 3-7 with the simple evaporation expression (dashed curves) confirms the main conclusions of the earlier studies: namely (1) an expression based on isotropic fragment emission from a moving nucleus and a constant nuclear temperature can fit the $90^{\circ}$ data near the yield maximum, but (2) there is more forward peaking in the fragment emission than can be explained even after correction for center-of-mass motion of the emitting nucleus and (3) the effective Coulomb barrier is about $40-50$ percent of that calculated by a classical tangent spheres estimate.

\section{Evaporation Spectra Based on a Maxwellian Distribution in Excitation Energy}

In Refs. $I$ and 2 it was difficult to fit the entire range of energy in the measured energy spectra for $\mathrm{Li}, \mathrm{Be}$, and $\mathrm{B}$ with the evaporation expression based on constant nuclear temperature and it was noted that the changing slope of the high energy portions of the spectrum could be interpreted to require a steady increase in nuclear temperature to a value of $20 \mathrm{MeV}$ or higher. It is 
reasonable to expect a range of excitation energies for the collection of struck nuclei from the conventional description of the initial high-energy cascade. In principle, one might hope to determine the distributions in excitation energy by examining the output from some detailed Monte-Carlo calculations of the high energy cascade of the type done by Chen et al. ${ }^{7}$ or Bertini et al. ${ }^{8}$ This can be done for proton bombarding energies of several hundred MeV but is inexact for $\mathrm{GeV}$ protons because of incomplete information on inelastic collisions for the proton-nucleon interactions needed as input for the cascade calculations. Even with reliable input data the calculation is a major undertaking and has not been done up to now except for pioneering attempts by Metropolis et al. 9 and Bertini et al. 8

In the absence of a theoretical model, we decided it would be interesting to explore the influence of an arbitrary distribution in excitation energy which follows our intuition, as well as the preliminary indications from the cascade calculations, as to its form: namely, that it rise rapidly to some most probable value and then decrease slowly out to high values. A Maxwellian distribution fits this description and was chosen for its many mathematical advantages.

We programmed a new calculation, referred to as MAXVAP in the remainder of this paper, in which the same formula as used in the above constant $\tau$ model was used for the evaporation of particles. This was taken from LeCouteur's treatment $^{10}$ of the Weisskopf evaporation theory. The new feature introduced in the MAXVAP calculation was the incorporation of a Maxwellian excitation energy distribution. Another important feature introduced in the MAXVAP calculation was the assumption that the velocity of the struck nucleus $\mathrm{v}$ was directly proportional to excitation energy, $E^{*}$. The details of this model are given 
in Appendix C. Although it is known from published calculations of the cascade step that there is a distribution in atomic charge and mass number for excited nuclei present at the end of the initial cascade step, we did not complicate the MAXVAP program by introducing this additional distribution, but we selected an average emitting nucleus somewhat lower in $\mathrm{Z}$ and $\mathrm{A}$ than the target nucleus. To facilitate comparison with the earlier work we used the same choices: namely ${ }^{96} \mathrm{TC}$ as the emitting nucleus representative of the $\mathrm{Ag}$ target and ${ }^{220} \mathrm{Rn}$ as representative of the $U$ target. The selection of $96_{\mathrm{TC}}$ as the emitting nucleus for a $\mathrm{Ag}$ target is reasonable on the basis of the calculation of Bertini et al. ${ }^{8}$ of $3 \mathrm{GeV}$ protons on ${ }^{100} \mathrm{Ru}$. In Refs. I and 2 it was stated that sample calculations confirmed that the predicted properties of emitted fragments are not sensitive to changes of several units in $\mathrm{Z}$ or $\mathrm{A}$ of the emitting nucleus. The results of the MAXVAP calculation are illustrated by the solid curves in Figs. 3-10。

There are only three input parameters in the calculation; the most probable excitation energy, $\mathrm{E}_{\mathrm{m}}{ }^{*}$, the fraction of the nominal Coulomb barrier, $k$, needed to fit the maximum of the energy distribution, and the level density parameter given by $a=M / b$, where $M$ is the mass number and $b$ is an adjustable constant. In the constant temperature evaporation model. described in Appendix B it is necessary to specify two additional parameters; namely, the center of mass velocity, $\mathrm{v}$, and a parameter $\mathrm{n}$ which correlates $\mathrm{v}$ with $\mathrm{V}$, the velocity of the emitted fragment. In the MAXVAP model these parameters are unneeded because of the assumption that center of mass velocity is proportional to excitation energy •

Although there is no unique set of values for the three input parameters, a reasonable set was found to reproduce more or less well the characteristics of 
the low-energy fragments from $\mathrm{Ag}$. Generating families of curves to aid in the selection of the "best" parameter was easier for MAXVAP than for the 5 parameter expression of Appendix B。 A k value of 0.4 gave the best fit on the Coulomb barrier section of the spectrum and this value was relatively insensitive to variations in the $\mathrm{E}_{\mathrm{m}}{ }^{*}$ and level density parameters. On the other hand the calculated curves were quite dependent on the pairs of choices of the last two parameters. If $\mathrm{E}_{\mathrm{m}}^{*}$ was decreased, no change in the calculated curve was found if the level density was decreased at the same time. Best fits to the data were obtained with $\mathrm{E}_{\mathrm{m}}^{*}$ and level density pairs which corresponded to a nuclear temperature of about $11.5 \mathrm{MeV}$ evaluated from the expression, $\mathrm{E}_{\mathrm{m}}^{*}=a \tau^{2}$. The best fit of the $90^{\circ} \mathrm{Li}$ data were obtained with $\mathrm{k}=0.4$, $E_{m}^{*}=400 \mathrm{MeV}$ and $a=6(b=16)$ and these choices were used for all the curves drawn in Figs. 3-10. In each figure the curves were normalized to the $90^{\circ}$ data. In Figs. 3 and 4 there is clear improvement in fitting the $90^{\circ}$ data for ${ }^{6} \mathrm{Li}$ and ${ }^{7} \mathrm{Be}$ over that obtained with the constant temperature formula (dashed curves). This improvement is even more marked on the higher energy part of the spectrum discussed in Sec. IV. However, the MAXVAP expression shows the same defect as the constant temperature formula in its inability to account for a pronounced favoring of forward emission of the fragments in the center-of-mass system. No theoretical model now in the literature is able to account quantitatively for this feature of high energy reactions. MAXVAP curves for $B$ and $C$ are not significantly better than the constant temperature evaporation curves for the data shown in Figs. 6 and 7 . We may note also that the favoring of forward emission steadily decreases with $\mathrm{Z}$ until at oxygen the agreement of the data points with the curves is equally good at all 5 angles. An error in beam monitoring during the $135^{\circ}$ experiment probably accounts for the fact that the $135^{\circ}$ data for all fragments are high. 
IV. RESULTS AND DISCUSSION OF HIGH ENERGY DATA

A. Particle Identification

In the experiments performed with the detector telescopes and absorbers described in Sec. II.C, only the fragments of lowest atomic number had sufficient range to penetrate the $\mathrm{Al}$ absorber and the two $\Delta \mathrm{E}$ detectors. Figure 12 shows the particle spectrum obtained from the Ag target with telescope 2 (Table I) and the $690 \mathrm{mg} / \mathrm{cm}^{2}$ Al absorber. Figure 13 shows the spectrum obtained from the U target with telescope 4 and the $690 \mathrm{mg} / \mathrm{cm}^{2} \mathrm{Al}$ absorber.

\section{B. Energy Spectra for $\mathrm{Li}, \mathrm{Be}, \mathrm{B}$ and C}

Energy data for ${ }^{6} \mathrm{Li}$ and ${ }^{7} \mathrm{Li}$ obtained in the runs with four different thicknesses of aluminum absorber are shown in Fig. 14. The solid lines show the trend of the data and have no theoretical significance. In the case of the $\mathrm{Ag}$ target data, these lines show the extension of the $20^{\circ}$ data of Fig. 3 , with the difference that Fig. 3 includes all lithium isotopes, whereas Fig. 14 shows ${ }^{6} \mathrm{Li}$ and ${ }^{7} \mathrm{Li}$ contributions, separately. The dashed lines represent curves computed by the MAXVAP code for the following parameter choices: $E_{m}^{*}=400 \mathrm{MeV}$, $k=0.40$ and $a=6.0(b=16)$. The curve is the same as that shown in Fig. 3 , except for a scale adjustment required by the fact that individual isotopes are displayed in Fig. 14. The MAXVAP curve lies below the $20^{\circ}$ data at all energies but has the same slope out to about $90 \mathrm{MeV}$. On the other hand, the constant temperature evaporation expression of Appendix B generates a curve dropping steeply away from the data in the 60-90 MeV region. Above $100 \mathrm{MeV}$, however, there is a sharp break in the energy spectrum and the appearance of a distinct high-energy "tail" which cannot be represented by the evaporation calculation even with a maxwellian distribution of temperatures. If we examine the $U$ target data we notice the same sharp break in the spectrum above $100 \mathrm{MeV}$. 
Similar comments may be made about the ${ }^{7} \mathrm{Be}$ data which are plotted in Fig. 15. In this figure we have included the low energy points for Ag near the Coulomb barrier and a dashed curve showing the MAXVAP computation. For the Ag data, the entire range of energy from 10 to $140 \mathrm{MeV}$ is reasonably well reproduced by the evaporation calculation for an $\mathrm{E}_{\mathrm{m}}{ }^{*}$ value of $400 \mathrm{MeV}$. However, if the MAXVAP calculation is normalized to the $90^{\circ}$ data as shown in Fig. 4, the $20^{\circ}$ data points lie well above the calculated curve for $20^{\circ}$. Beyond $140 \mathrm{MeV}$ there is a distinct break in the spectrum revealing a high energy tail extending, at least to $400 \mathrm{MeV}$.

We have considered the possibility that the high energy component in the Li and Be spectra could be caused by some background or instrumental effect. We believe the observed effect is real. A strong argument to support this is that particle identification was made on those fragments after penetration of the thick absorbers. In all the experiments particle identification spectra similar to those shown in Figs. 12 and 13 were obtained and only the data appearing in the well defined $\mathrm{Li}$ or ${ }^{7} \mathrm{Be}$ peaks were selected for plotting. We believe that the criteria set on the data are so stringent that there can be no doubt of the reality of the high energy component extending up to $300 \mathrm{MeV}$ for $\mathrm{Li}$ and to $400 \mathrm{MeV}$ for $7_{\mathrm{Be}}$.

These high energy fragments cannot be described by any version of evaporation theory we have considered so far. In all likelihood they are not produced by evaporation but by some process occurring during the cascade. If so, they should be very strongly peaked in the beam direction. We regret we did not have sufficient beam time to make long runs at angles other than $20^{\circ}$ in order to determine whether the angular distribution of 200 to $400 \mathrm{MeV}$ fragments favors the forward direction much more strongly than that of fragments of lower energy, as known already from Refs. 1 and 2 and the present study. 


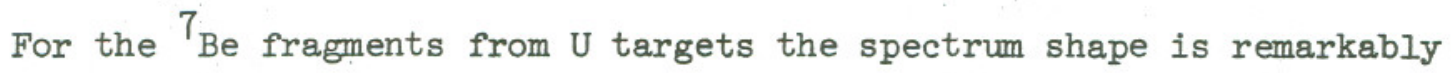
similar except for the shift in the location of the turnover near the Coulomb barrier. It is possible to generate a curve from the MAXVAP calculation which tracks the data points out to about $120 \mathrm{MeV}$ before the transition into the high energy tail. However, the $\mathrm{E}_{\mathrm{m}}{ }^{*}$ value necessary to achieve this agreement is greater than $2000 \mathrm{MeV}$ when ${ }^{220} \mathrm{Rn}$ is taken as a representative evaporating nucleus. This is an unphysically high value for $\mathrm{E}_{\mathrm{m}}{ }^{*}$ because the average value of excitation would approach the bombarding energy and from all we know about $\mathrm{GeV}$ proton interactions with uranium the average excitation must be much lower than this. If we assume that $7_{B e}$ is evaporated from a fission or fragmentation product of $U$ with about half the charge and mass of $U$, the $\mathrm{E}_{\mathrm{m}}{ }^{*}$ value can be reduced to $500 \mathrm{MeV}$. However, then the high energy fragment distribution would be isotropic in disagreement with the experimental fact.

We now consider the data for the heavier isotopes of Be shown in Fig. 16 and for B and C shown in Figs. 17 and 18. Because of the lower yield of these particles, a measurable number of them were observed only in the runs with the thinnest of the 4 aluminum absorbers, and it was not possible to determine whether the energy spectra of these fragments had high-energy components (tails) similar to those observed for ${ }^{6} \mathrm{Li},{ }^{7} \mathrm{Li}$, and ${ }^{7} \mathrm{Be}$. A MAXVAP calculation with an $\mathrm{E}_{\mathrm{m}}^{*}$ value of $400 \mathrm{MeV}$ agrees roughly with the ${ }^{9,10} \mathrm{Be}$ data from $\mathrm{Ag}$ out to $140 \mathrm{MeV}$ and with the $\mathrm{B}$ data out to $180 \mathrm{MeV}$. However, in the case of the U target data, we encounter the same difficulty that the MAXVAP curve agrees with the data only if a much higher value of $\mathrm{E}_{\mathrm{m}}^{*}$ is chosen, namely $\mathrm{E}_{\mathrm{m}}^{*}=2000 \mathrm{MeV}$. We compared our high-energy data on $\mathrm{Be}, \mathrm{B}$, and $\mathrm{C}$. fragments from uranium with those appearing in Ref. 1 in the energy region where they overlapped 
and found our cross section to be a factor of 2 lower. ${ }^{11}$ On the other hand, our cross section data on Li agree with the previously published data within the experimental error of about 25 percent.

\section{High Energy Data for He Isotopes}

In Refs. 1 and 2 it was observed that the high energy part of the $3_{\mathrm{He}}$ spectrum was flatter than that for ${ }^{4} \mathrm{He}$ and that above a fragment energy of about $100 \mathrm{MeV}$ the ${ }^{3} \mathrm{He}$ yield was greater than that of ${ }^{4} \mathrm{He}$. Alard ${ }^{12}$ has also noted a high $3 \mathrm{He} /{ }^{4} \mathrm{He}$ ratio in fragments from $\mathrm{Al}$ bombarded with $20 \mathrm{GeV}$ protons and in C, Al, Au targets bombarded with $600 \mathrm{MeV}$ protons. Piroué and Smith ${ }^{13}$ measured high energy $3_{\mathrm{He}}$ produced in Pt bombarded with $2.9 \mathrm{GeV}$ protons.

In our own research the measurement of He spectra was not a primary goal and the detector telescopes were not optimum for this purpose. Nonetheless, the $\mathrm{He}$ isotopes were clearly resolved in some of the thick absorber experiments (see Figs. 12 and 13) and a few points were determined in the high energy part of the energy spectra at $20^{\circ}$ for ${ }^{3} \mathrm{He},{ }^{4} \mathrm{He}$, and ${ }^{6} \mathrm{He}$. Figure 19 shows the results for the Ag target together with previous data from Ref. 2. Our ${ }^{4}$ He points do not join smoothly with the Ref. 2 data; our cross section is a factor of 2 lower in the overlap region near $100 \mathrm{MeV}$. A discrepancy exists also in the ${ }^{3} \mathrm{He}$ data but it is somewhat less for this isotope. In the case of ${ }^{6}$ He the overlap is smooth within the experimental error. The dislocation of the points for ${ }^{3} \mathrm{He}$, ${ }^{4} \mathrm{He}$, and ${ }^{6} \mathrm{He}$ in the $\mathrm{Ag}$ target run with the $690 \mathrm{mg} / \mathrm{cm}^{2}$ aluminum absorber convince us there was a factor of 2 error in the monitoring or in the processing of the data for that run. We adjusted these points downward as shown by the arrows, and applied the same adjustment to the ${ }^{6} \mathrm{Li},{ }^{7} \mathrm{Li}$, and ${ }^{7} \mathrm{Be}$ data obtained in the same run and displayed in Figs. 14 and 15. 
In the case of $U$ we can report the following cross sections at $20^{\circ}$ : for ${ }^{4} \mathrm{He}$ energies of 78,100 , and $135 \mathrm{MeV}$ the cross sections are 600,300 , and $125 \mu \mathrm{b} \mathrm{MeV}{ }^{-1} \mathrm{Sr}^{-1}$, respectively. For ${ }^{6} \mathrm{He}$ energies of 95,117 , and $160 \mathrm{MeV}$ the cross sections are 20,8 , and $3.5 \mathrm{\mu b} \mathrm{MeV}^{-1}, \mathrm{Sr}^{-1}$, respectively. The ${ }^{4} \mathrm{He}$ data of Ref. 1 extend to $120 \mathrm{MeV}$ and at this energy the Ref. 1 cross section is double the one reported here. The ${ }^{6}$ He data in the two studies agree within 20 percent up to $120 \mathrm{MeV}$ where the Ref. I data cut off.

The presence of a strong ${ }^{\text {He contribution to the high energy He }}$ spectrum is a striking and interesting phenomenon which is discussed in detail by Alard ${ }^{12}$ in a thesis which reviews the literature on the observation of energetic deuterons, tritons, and $3_{\mathrm{He}}$ in high energy reactions and the explanations proposed for their production. Some theoretical papers propose the direct creation of deuterons and mass-3 particles in the interaction of the incident proton with a target nucleon, ${ }^{14}$ while others propose the formation of these particles out of the cloud of cascade neutrons and protons when 2 or more of these are produced with small relative momenta. ${ }^{15-17}$ Still others apply new statistical thermodynamic concepts to the calculation of particle production from a volume of hadrons strongly heated by the intranuclear cascade. ${ }^{12,18}$ 
V. SUMMARY

This paper describes new experimental data on energy spectra of identified fragments from $\mathrm{Ag}$ and $\mathrm{U}$ targets bombarded with $5.0 \mathrm{GeV}$ protons. It may be considered an extension of the work described in 2 previous publications. ${ }^{1,2}$ For Ag targets, segments of the energy spectra of light elements up to sodium were measured at 5 angles to the beam with a detector telescope containing a $16 \mu \mathrm{m} \Delta \mathrm{E}$ detector. For both $\mathrm{Ag}$ and $\mathrm{U}$ targets the high energy part of the spectrum was measured at $20^{\circ}$ to the beam for $\mathrm{He}, \mathrm{Li}, \mathrm{Be}, \mathrm{B}$, and C fragments with telescopes including thicker detectors and absorbers. Two evaporation models were tested to determine whether they could explain the main features of the light element spectra, with the most severe tests posed by the data on $\mathrm{Li}$ and $\mathrm{Be}$. A simple evaporation expression based on a fixed nuclear temperature for the evaporating nucleus can reproduce the shape of the spectra only in the region of the maximum. This maximum defines an effective Coulomb barrier. At the higher energy, particularly above $100 \mathrm{MeV}$ for $\mathrm{Li}$ and $\mathrm{Be}$, the calculations in no way reproduce the slope of the data. A modified evaporation calculation called MAXVAP which involves an arbitrary Maxwellian distribution in excitation energy can reproduce the data rather well out to about $100 \mathrm{MeV}$ for $\mathrm{Li}$ and Be fragments from $\mathrm{Ag}$ with acceptable choices of excitation energy and level density parameters. $\left(E_{m}^{*}=400 \mathrm{MeV}\right.$, $a=6.0)$. For fragments from $U$ the MAXVAP calculations reproduces the $20^{\circ}$ data reasonably well out to the same energy but only by use of a most probable excitation energy of $2 \mathrm{GeV}$ which seems much too high, or by use of a level density parameter $\mathrm{b} \sim 80$ which seems unreasonable.

Both evaporation models have difficulty in explaining the relative intensities of the particle emission at the 5 angles studied. Even after 
correction for center-of-mass motion the data indicate a pronounced favoring of forward emission. This favoring decreases with atomic number and seems to disappear for oxygen and higher elements. Another curious feature, independent of the chosen evaporation expression, is the fact that the effective Coulomb barrier for fragment emission in these high energy reactions is only 40 to 50 percent that estimated from a simple tangent spheres model. An effect contributing to this reduction in the Coulomb barrier is discussed by Moretto. 25 In the case of ${ }^{6} \mathrm{Li}, 7_{\mathrm{Li}}$, and ${ }^{7} \mathrm{Be}$ the $20^{\circ}$ data clearly reveal a highenergy component which cannot be described by the MAXVAP calculation and must represent fragments produced in some little understood way during the initial high energy cascade. This statement applies to ${ }^{6} \mathrm{Li}$ fragments measured in this study between 120 and $320 \mathrm{MeV}$ and ${ }^{7}$ Be fragments between 120 and $420 \mathrm{MeV}$. The He isotope yields as measured out to $\sim 160 \mathrm{MeV}$ show $3{ }^{3 e} /{ }^{4} \mathrm{He}$ ratio exceeding 1.0. This also is probably a consequence of the cascade step. Some suggestions for possible mechanisms for production of very high energy light fragments which have appeared in the literature, are cited but not discussed.

In the process of working up $\Delta \mathrm{E} \times \mathrm{E}$ data which included information on many particles over wide energy ranges the necessity for a better method of extraction of particle identity arose and this need was met by the method described in Appendix A. 


\section{ACKNOWLEDGMENTS}

We acknowledge with thanks the many contributions of the operating crew. and staff of the Bevatron to the success of these experiments. We are indebted to the semiconductor development group of LBL for fabrication of many of the detectors and to Gordon Steers for preparation of the $1 \mathrm{mg} / \mathrm{cm}^{2}$ silver target. We owe thanks to our colleagues Arthur M. Poskanzer, Gilbert W. Butler, and J. David Bowman, and Richard E. Eppley for advice and assistance in the experimental and data analysis parts of the study. We are specially indebted to Arthur Poskanzer for a critical reading of the manuscript. The The Canadian members of the team of authors wish to express their thanks to the Lawrence Berkeley Laboratory for its hospitality and support. 


\section{APPENDIX A: NEW TREATMENT OF $\triangle E / \triangle X$ AND E DATA TO ACHIEVE IMPROVED PARTICLE IDENTIFICATION}

\section{A. Previous Treatments}

Semiconductor detector telescopes consisting of a thin transmission detector to measure rate of energy loss $(\Delta \mathrm{E})$ and a thicker detector to measure the residual energy $E$ have seen widespread use in recent years for the identification of charge and mass of the products of nuclear reactions. Severai methods of treating the $\Delta \mathrm{E}$ and $\mathrm{E}$ information to achieve this purpose have been applied. One method uses the relationship ${ }^{19}(\mathrm{E}+\Delta \mathrm{E}) \times(\Delta \mathrm{E}) \alpha \mathrm{AZ}{ }^{2}$ which is an approximation to the Bethe-Block formula for the rate of energy loss of a charged particle. Another ${ }^{3}$ is based on the empirical fact that the ranges of light nuclei follow a power-low relationship of the form $R=a E^{b}$ where $R$ is the range of a particle of energy $\mathrm{E}$, a is a constant which is characteristic of the particle and the exponent $\mathrm{b}$ is approximately equal to 1.6 but varies somewhat with $\mathrm{Z}$. For particles with energy in the range well described by this power law it can be shown that the function $(E+\Delta E)^{b}-E^{b}=T / a$, where $T$ is the thickness of the $\Delta \mathrm{E}$ detector and quantity $\mathrm{T} / \mathrm{a}$ is a constant characteristic of a specific particle. The calculation of this power law function can be done by the analog circuitry designed originally by Goulding and Landis ${ }^{3}$ or it can be done in a computer by manipulation of the digital record of the $E$ and $\Delta E$ pulse heights. The latter offers the advantage that parameter $\mathrm{b}$ can be varied until the best particle resolution is achieved.

The above treatments provide satisfactory resolution of the particle spectrum for experiments in which the number of species and the range of energies for each species are limited. However, in studies of high energy reactions where a great number of different species, each covering a wide energy range is present, the particle resolution is not completely satisfactory 
as is made evident in two dimensional plots of the particle identification parameter versus total energy of the particle. Butler, Poskanzer, and Landis ${ }^{18}$ revised the treatment based on the power-law relationship and introduced an improved algorithm of the form

$$
\left[\left(\frac{E+E}{k}\right)^{m}-\left(\frac{E}{k}\right)^{m}\right]^{1 / 2},
$$

where the exponent $\mathrm{m}$ is not $\mathrm{b}$ but $\mathrm{b}-\mathrm{c} \Delta \mathrm{E} / \mathrm{T}$. The second term is a correction on b where $C$ has a typical value of 0.05 in units of $\mathrm{mg} / \mathrm{cm}^{2}-\mathrm{MeV}$ and $\mathrm{T}$, as before, is the $\Delta E$ thickness in $\mathrm{mg} / \mathrm{cm}^{2}$. The divisor $\mathrm{k}$ is set equal to 300 but its exact value is not critical. This algorithm is applied by reading the $\Delta E$ and $\mathrm{E}$ information from magnetic tape and performing the algebra in a computer. A range of choices of $b$ and $c$ can be tried until the best particle resolution is achieved.

This treatment of Butler, et $a l .^{20}$ is a considerable improvement on the other methods cited above for particle identification in a complex mixture. We found it to be quite satisfactory for the analysis of the high energy data in the present study. However, in the case of the data taken. with the $16 \mu \Delta \mathrm{E}$ detector, we found it was possible to get a more satisfactory resolution with the method now to be described.

\section{B. New Treatment}

The Bethe-Block equation 21,22 can be written in the form

$$
\mathrm{MZ}^{2} \propto \frac{\left(\mathrm{E}_{\mathrm{T}}\right)(\Delta \mathrm{E})}{\ln \mathrm{E}_{\mathrm{T}}+\text { const. }} .
$$


It is customary to ignore the $\ln E_{T}$ term, but we decided to retain it since we observed the $E_{T}$ vs $\Delta E$ data to follow an exponential form (see Fig. 20). We arbitrarily ignored the constant in the denominator and after several tests of. Eq. (A-2) and minor variants with our data we chose the following expression as our favored algorithm,

$$
P I=k\left[\left(\frac{E_{T}}{\ln \bar{E}_{T}}\right)\left(\frac{\Delta E}{1024}\right)^{b}\right]^{1 / 2},
$$

where $\mathrm{k}=\left(\frac{\ln 1024}{1024}\right)^{1 / 2}(1024)$. This constant and the divisor 1024 in A-3 are normalization factors related to the fact that the input pulses were digitized on a scale of 1024. The square root was taken so that the PI scale would be linear in $\mathrm{Z}$ instead of $\mathrm{z}^{2}$ and the exponent $\mathrm{b}$ was introduced to correct for neglect of the constant in A-2. Generally, b has the value 1.0 for the lightest elements ( $\mathrm{He}, \mathrm{Li}, \mathrm{Be}$ ) and increases exponentially with $\mathrm{Z}$ to a value of about 10 for $\mathrm{Z}=18$. A fixed value of $\mathrm{b}$ results in good particle resolution over a range of several elements. Inclusion of the $\ln \mathrm{E}_{\mathrm{T}}$ term holds PI constant over a wide range of $\mathrm{E}_{\mathrm{T}}$ and use of a variation in $\mathrm{b}$ provides uniform particle resolution over a range of $\mathrm{Z}$.

\section{Procedure in Use of New Algorithm}

For each run the tape record of digitized $\Delta \mathrm{E}$ and $\mathrm{E}_{\mathrm{T}}$ pulse heights on an event-by-event basis was read into the computer and the data were summed into a $E_{T}$ vs $\Delta E$ matrix of dimensions $1024 \times 1024$. This matrix was written on a magnetic tape which was used as the source tape for subsequent data manipulation. This was the most time consuming step in the whole process. (For $10^{6}$ events it required about 10 minutes of CDC-6600 central processor time.) In the case of data from 3-element telescopes the two $\Delta \mathrm{E}$ values were summed. A 
sample result of the summation of the data in $E_{T}$ vs $\Delta E$ form is shown in topographic map form in Fig. 20 but with the data compressed into a $128 \times 128$ matrix.

A computer program, PICAL, using the $\mathrm{E}_{\mathrm{T}}$ vs $\Delta \mathrm{E}$ matrix as input, calculated a PI value for each element in the matrix making use of the expression (A-3) and a preselected value of the exponent $\mathrm{b}$. This procedure converted the $\mathrm{E}_{\mathrm{T}}$ vs $\triangle \mathrm{E}$ matrix into an $\mathrm{E}_{\mathrm{T}}$ vs $\mathrm{PI}$ matrix. The program generated a particle spectrum plot by projecting all data on to the PI axis. This process was repeated for other values of the exponent $b$ and the particle plots were inspected to determine which values of $\mathrm{b}$ were best for each section of the PI scale. A final calculation was then made with different $b$ values applied to different regions of the data matrix and a final $E_{T}$ vS PI matrix and a final particle spectrum were generated. Figure 21 illustrates the influence of the $b$ parameter on the particle resolution, including the use of multiple values of $\mathrm{b}$ in a final processing of the data. Figures 1 and 2 are also examples of final particle spectra obtained by use of multiple values of b. Figure 22 is a plot of the final $\mathrm{E}_{\mathrm{T}}$ vs PI matrix in topographical map form. Some details are lost in this representation because the $1024 \times 1024$ scale was reduced to $128 \times 128$ in the illustration. The success of the PI algorithm is measured by the straightness of the ridges (i.e. no variation in PI with increase in $E_{T}$ ).

By inspection of the final particle spectrum it was possible to select PI channel numbers to isolate the energy data belonging to a specific particle type. The use of such PI "windows" in the reduction of the energy data is described in Sec. III.E. 
APPENDIX B: CONSTANT TEMPERATURE EVAPORATION EXPRESSION ${ }^{*}$

The energy spectrum in the moving system of the evaporating nucleus is represented by the expression

$$
P(\varepsilon)=\sum_{k=\langle k\rangle-\Delta}^{\langle k\rangle+\Delta}(\varepsilon-k B) e^{-(\varepsilon-k B) / \tau}, \varepsilon>k B,
$$

where $\varepsilon$ is the disintegration energy, $B$ is the nominal Coulomb barrier and $\mathrm{kB}$ is the effective Coulomb barrier. $\Delta$ is a small parameter which specifies the spread in $k$ values caused by the fact that a variety of emitting nuclei are involved. The energies of fragments of mass A emitted by a nucleus of mass $M$ were corrected for recoil of the residual nucleus and related to a velocity $\mathrm{V}$ in the moving system by the equation

$$
(1-\mathrm{A} / \mathrm{M}) \varepsilon=1 / 2 \mathrm{mV}^{2} \text {. }
$$

At $90^{\circ}$ the laboratory energy $E$ was taken equal to $(1-\mathrm{A} / \mathrm{M}) \varepsilon$. At the other angles the laboratory frame velocity $V_{L}$ was corrected for the velocity of the moving system $\mathrm{v}$, which was typically about 0.006 times the speed of light. The double differential laboratory cross sections were calculated from $P(\varepsilon)$ by use of the relation

$$
\frac{\partial^{2} \sigma}{\partial E \partial \Omega}=P(\varepsilon) \frac{E}{\varepsilon} \frac{d \varepsilon}{d E}
$$

\footnotetext{
${ }^{*}$ See Ref. I for more complete description.
} 
which is proportional to $\mathrm{P}(\varepsilon) \sqrt{\mathrm{E} / \varepsilon} /\left(\mathrm{dV}_{\mathrm{L}} / \mathrm{dV}\right)$. A positive correlation between $\mathrm{V}$ and $\mathrm{v}$ was introduced to fit the data. It allows for the fact that fragments which are emitted from a struck nucleus moving with greater than average velocity will be ejected with greater than average velocity in the moving system. The correlation takes the form

$$
\frac{v-\langle v\rangle}{\langle v\rangle}=n \frac{v-\langle v\rangle}{\langle v\rangle}
$$

where the average quantity $(V)$ was taken to be the root mean square $V$ obtained from the average energy $\langle\varepsilon\rangle$, which is equal to $\langle k\rangle B+2 \tau$ for a Maxwellian distribution. 


\section{APPENDIX C: AN EVAPORATION CALCULATION BASED ON A MAXWELLIAN DISTRIBUTION IN EXCITATION ENERGY}

A new expression for calculation of fragment evaporation spectra was devised in an effort to allow for the fact that fragment emission in nuclear reactions induced by $\mathrm{GeV}$ protons occurs from nuclei with a wide distribution in excitation energy. In the absence of clear theoretical guidance on the form of this distribution, a Maxwellian distribution of excitation energies was chosen, as mentioned in the main text Sec. III.C. In addition, the forward momentum of the emitting nucleus and the appropriate kinematics were included in a more straightforward way than in the calculation described in Appendix $B$. This new calculation, called MAXVAP, was developed as follows.

The forward momentum of the emitting nucleus was calculated for each value of excitation energy by the expression

$$
\mathrm{P}_{11} / \mathrm{P}_{\text {inc }}=\mathrm{E}^{*} / \mathrm{E}_{\max }^{*},
$$

obtained from Monte Carlo ${ }^{7}$ calculations and some experimental studies. ${ }^{23}$ The maximum incident momentum, $\mathrm{P}_{\text {inc }}$, was determined from the incident energy of the bombarding proton by relativistic expressions. The maximum excitation energy, $\mathrm{E}_{\text {max }}^{*}$, was set equal to the incident energy minus the small amount associated with the forward momentum of the hypothetical compound nucleus. The velocity of the emitting nucleus, commonly referred to as $\mathrm{v}$, can then be calculated by the expression:

$$
v=E^{*}\left(\frac{2 E_{0}}{T}+1\right)^{1 / 2}\left(1+A_{t}\right) / M_{c} A_{t}
$$

where $\mathrm{E}^{*}$ is the excitation energy, $\mathrm{E}_{0}$ is the rest mass of the proton, $\mathrm{T}$ is the incident proton energy, $A_{t}$ is the mass of the target nucleus, $M$ is the mass of 
the emitting nucleus, and $c$ is the velocity of light. For a given set of conditions, $\mathrm{v}=$ const $\times \mathrm{E}^{*}$.

We assumed an isotropic distribution of the evaporated fragments in the center of mass system. To compare the predictions with our experimental results, we transformed the predicted energy distributions into the laboratory system by means of the non-relativistic relationship 24

$$
\mathrm{V}=\mathrm{v} \cos \theta+\left(\mathrm{v}_{\mathrm{c}}^{2}-\mathrm{v}^{2} \sin ^{2} \theta\right)^{1 / 2}
$$

where $\mathrm{V}$ is the velocity of the emitted particle at an angle $\theta$ in the laboratory system, and $V_{c}$ is the velocity of the particle in the center of mass system. $V$ is given by $V^{2}=2 E / A$ where $E$ is the measured energy for the particle of mass $A . V_{c}$ is obtained from $v_{c}^{2}=2 E_{c} / A$ where $\mathrm{E}_{\mathrm{C}}$ is the energy of the particle in the center of mass system. However, this center of mass energy must be corrected for the recoil of the emitting nucleus to obtain the true disintegration energy, $\varepsilon$. The final expression for $\mathrm{V}_{c}$ is then $\mathrm{V}_{\mathrm{c}}^{2}=2 \varepsilon\left(\frac{\mathrm{M}-\mathrm{A}}{\mathrm{MA}}\right)$. It is then possible to calculate $\varepsilon$ in terms of measured or calculable quantities by rearranging Eq. C-3 to give:

$$
\varepsilon=\frac{M A}{2(M-A)}\left[(v-v \cos \theta)^{2}+v^{2} \sin ^{2} \theta\right] .
$$

The correction for the transformation of the angular distribution from the center of mass to the laboratory system was calculated by 24

$$
\begin{aligned}
\frac{\sigma_{L}(\theta)}{\sigma_{c}(\theta)} & =\frac{v^{2}}{V_{c}\left(v_{c}^{2}-v^{2} \sin ^{2} \theta\right)^{I / 2}} \\
& =\frac{v^{2}}{(V-v \cos \theta)\left(v^{2}+v^{2}-2 V v \cos \theta\right)},
\end{aligned}
$$


which is again in terms of known quantities.

To recapitulate, the new calculation starts with the same LeCouteur formula ${ }^{10}$ for evaporation of particles as used in the constant $\tau$ model,

$$
P(\varepsilon) \mathrm{d} \varepsilon=\frac{\varepsilon-\mathrm{kB}}{\tau^{2}} \exp \left(-\frac{\varepsilon-\mathrm{kB}}{\tau}\right) \mathrm{d} \varepsilon
$$

The Maxwellian excitation energy distribution (C-7), in which $\mathrm{E}_{\mathrm{m}}^{*}$ is the most probable excitation energy, was folded into C-6 by summing over $E^{*}$ for each value of $\mathrm{E}$

$$
P\left(E^{*}\right)=E^{*} \exp \left(-E^{*} / E_{m}^{*}\right)
$$

In addition the velocity of the struck nucleus, $\mathrm{v}$, was made proportional to $\mathrm{E}^{*}$. The resulting expression is:

$$
\mathrm{P}\left(\varepsilon, \mathrm{E}^{*}\right)=\mathrm{a}(\varepsilon-\mathrm{kB}) \exp -\left[\left(\frac{\varepsilon-\mathrm{kB}}{\left(\mathrm{E}^{*} / \mathrm{a}\right)^{1 / 2}}\right)+\frac{\mathrm{E}^{*}}{\mathrm{E}_{\mathrm{m}}^{*}}\right] \text {, }
$$

where $\mathrm{E}^{*}=a \tau^{2}$ and $\mathrm{a}$ is the level density parameter given by $\mathrm{M} / \mathrm{b}$. By calculating the value of $\varepsilon$ from Eq. $\mathrm{C}-4$ at each value of $\mathrm{E}$, we obtained an expression for the emission probability in terms of $\mathrm{E}$ and $\mathrm{E}^{*}$. The laboratory cross section was then calculated by the expression:

$$
\frac{d \sigma^{2}}{d E d \Omega} \propto \sum_{E^{*}=1}^{E_{\max }^{*}} P\left(E, E^{*}\right) \frac{\sigma_{L}(\theta)}{\sigma_{c}(\theta)} .
$$

The upper limit on this summation represents a truncation of the high end of the Maxwellian energy distribution, which is not serious unless very high $\mathrm{E}_{\mathrm{m}}^{*}$ values are used. 


\section{FOOTNOTES AND REFERENCES}

*Work supported in part by the U. S. Atomic Energy Commission and in part by the National Research Council of Canada.

1. A. M. Poskanzer, G. W. Butler, and E. K. Hyde, Phys. Rev. C 3, 882 (1971).

2. E. K. Hyde, G. W. Butler, and A. M. Poskanzer, Phys. Rev. C 4 , 1759 (1971).

3. F. S. Goulding, D. A. Landis, J. Cerny, III, and R. H. Pehl, Nucl. Instr. Methods 31, I (1964); F. S. Goulding, D. A. Landis, J. Cerny, III, and R. H. Pehl, IEEE Trans. Nucl. Sci. 13, 514 (1966).

4. C. C. Maples, Jr. and J. Cerny, unpublished (1966-8).

5. P. G. Steward, Lawrence Radiation Laboratory Report UCRL-18127, May 1968.

6. H. Bichsel and C. Tschalaer, Nucl. Data A3, 343 (1967).

7. K. Chen, Z. Fraenkel, G. Friedlander, J. R. Grover, J. M. Miller, and Y. Shimamoto, Phys. Rev. 166, 949 (1968).

8. Hugo W. Bertini and Miriam P. Guthrie, Nucl. Phys. Al69, 670 (1971).

9. N. Metropolis, R. Bivens, M. Storm, A. Turkevich, J. M. Miller, and G. Friedlander, Phys. Rev. 110, 185 (1958); 110, 204 (1958).

10. K. J. LeCouteur, Proc. Phys. Soc. (London) A63, 259 (1950).

11. In the course of this comparison we found that the ${ }^{11_{C}}$ and ${ }^{15} \mathrm{C}$ data in Fig. 11 Ref. 1 are plotted a factor of 10 too high. Also in Fig. 16 of Ref. 2 there is a scale error in the plotting of the $20^{\circ}$ and $90^{\circ}$ data for $7_{\mathrm{Be}}$.

12. J. P. Alard, Thèse, Université de Clermont, France 1971.

13. P. A. Piroué and A. J. S. Smith, Phys. Rev. 148, 1315 (1966).

14. R. Hagedorn; Phys. Rev. Letters 5, 276 (1960).

15. C. Zupancic, Phys. Letters 4, 182 (1963).

16. A. Schwartzchild and C. Zupancic, Phys. Rev. 129, 854 (1963). 
17. S. T. Butler and C. A. Pearson, Phys. Rev. Letters 1, 69 (1961); 1, 77 (1962); Phys. Rev. 129, 836 (1963).

18. R. Hagedorn and J. Ranft, Suppl. Nuovo Cim. 6, 169, 298 (1968).

19. M. W. Sachs, C. Chasman, and D. A. Bromley, Nucl. Instr. Methods 41,213 (1966).

20. G. W. Butler, A. M. Poskanzer, and D. A. Landis, Nucl. Instr. Methods $\underline{89}$, $189(1970)$.

21. G. Dearnaley and D. C. Northrup, "Semiconductor Counters for Nuclear Radiations", $2^{\text {nd }}$ edition, E. and F. N. Sapon, Ltd., London (1966).

22. H. A. Bethe, Z. Physik 76, 293 (1932).

23. N. T. Porile, Phys. Rev. 120, 572 (1960).

24. J. B. Ball, Oak Ridge National Laboratory Report No. ORNI-3251 (1962).

25. L. G. Moretto, Lawrence Berkeley Laboratory Report LBL-634 (1972). 
Table I. Detector Telescope and Absorber Characteristics

\begin{tabular}{|c|c|c|c|c|c|c|}
\hline \multirow{2}{*}{ Telescope } & \multicolumn{4}{|c|}{ Detector thicknesses ( $\mu \mathrm{m})$} & \multirow{2}{*}{$\begin{array}{l}\text { Al absorber } \\
\text { thickness } \\
\left(\mathrm{mg} / \mathrm{cm}^{2}\right)\end{array}$} & \multirow{2}{*}{$\begin{array}{l}\text { Target } \\
\text { element }\end{array}$} \\
\hline & $\Delta \mathrm{E}_{2}$ & $\Delta \mathrm{E}_{1}$ & $E$ & $\mathrm{E}_{\mathrm{rej}}$ & & \\
\hline 1 & - & $16 \mathrm{SB}$ & 194 & 381 & 0 & $\mathrm{Ag}$ \\
\hline 2 & 100 & 61 & $1000 \mathrm{SB}$ & 381 & 35.7 & $\mathrm{U}, \mathrm{Ag}$ \\
\hline 2 & $"$ & $"$ & $"$ & $"$ & 432 & $\mathrm{Ag}$ \\
\hline 3 & 100 & 61 & $1524(\mathrm{Si}(\mathrm{Li})$ & 381 & 432 & $\mathrm{U}$ \\
\hline 3 & $"$ & $"$ & $"$ & $"$ & 1295 & $\mathrm{U}$ \\
\hline 4 & 135 & 96 & $2000 \mathrm{SB}$ & 381 & 690 & $\mathrm{U}, \mathrm{Ag}$ \\
\hline 4 & $"$ & $"$ & $"$ & . " & 1295 & $\mathrm{Ag}$ \\
\hline
\end{tabular}


Table II. Evaporation Curve Fitting Parameters

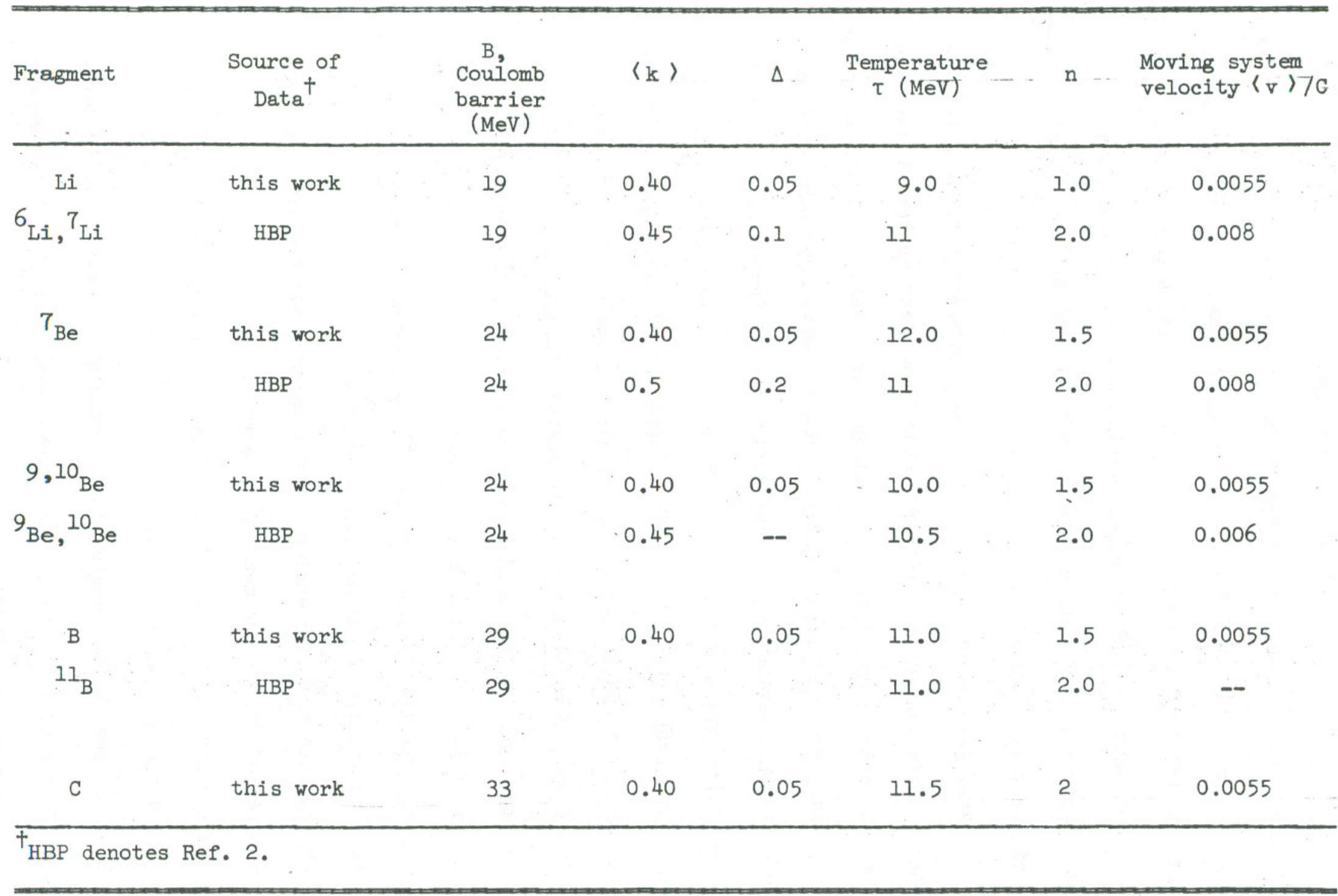




\section{FIGURE CAPTIONS}

Fig. 1. Spectrum of elements ejected at $20^{\circ}$ to beam direction in bombardment of $\mathrm{Ag}$ target with $5.0 \mathrm{GeV}$ protons. Fragments measured with $16 \mu \mathrm{m} \Delta \mathrm{E}$ detector and $194 \mu \mathrm{m} \mathrm{E}$ detector. Particle spectrum generated from $\Delta \mathrm{E}$ and E pulse heights by the technique described in Appendix A.

Fig. 2. Spectrum of elements ejected at $90^{\circ}$ to beam direction in bombardment of silver target with $5.0 \mathrm{GeV}$ protons.

Fig. 3. Energy spectra at 5 angles for lithium fragments ejected from Ag target. Vertical scale shift by factor 10 at each angle. Squares represent data from this study, dots represent data from Ref. 2. (Only a portion of the data from Ref. 2 are shown.) Dashed curve represents fitted curve calculated from simple evaporation theory (Appendix B) with fixed nuclear temperature normalized to $90^{\circ}$ data. Solid curves represent fitted curves from evaporation code MAXVAP (Appendix C) incorporating Maxwellian distribution of excitation energies, also normalized to $90^{\circ}$ data. $\mathrm{E}_{\mathrm{m}}^{*}=400 \mathrm{MeV}, \mathrm{k}=0.4$ and $\mathrm{a}=6$ in MAXVAP calculation.

Fig. 4. Energy spectra at 5 angles for ${ }^{7}$ Be ejected from $\mathrm{Ag}$ targets. See caption of Fig. 3 for meaning of curves.

Fig. 5. Energy spectra at 5 angles for ${ }^{9,10} \mathrm{Be}$ fragments ejected from $\mathrm{Ag}$ targets. See caption of Fig. 3 for meaning of curves.

Fig. 6. Energy spectra at 5 angles for B fragments ejected from Ag targets. See caption of Fig. 3 for meaning of curves.

Fig. 7. Energy spectra at 5 angles for $C$ fragments ejected from $\mathrm{Ag}$ targets. See Fig. 3 for meaning of curves.

Fig. 8. Energy spectra at 5 angles for $\mathrm{N}$ fragments ejected from Ag targets. Vertical scale shift by factor 10 at each angle. Solid lines represent evaporation expression MAXVAP. 
Fig. 9. Energy spectra at 5 angles for $\mathrm{Ne}$ fragments ejected from Ag targets. Vertical scale shift by factor 10 at each angle. Solid lines represent evaporation expression MAXVAP.

Fig. 10. Energy spectra at 5 angles for $\mathrm{Na}$ fragments ejected from Ag targets. Vertical scale shift by factor 10 at each angle. Solid lines represent evaporation expression MAXVAP.

Fig. 11. Energy spectra for elements from B through S ejected from Ag target at $20^{\circ}$ to the $5 \mathrm{GeV}$ proton beam direction.

Fig. 12. Particle spectrum of fragments ejected at $20^{\circ}$ to beam direction in bombardment of Ag target with $5.0 \mathrm{GeV}$ protons. Fragments penetrating Al absorber $\left(690 \mathrm{mg} / \mathrm{cm}^{2}\right)$ were measured with 3-element telescope (see Table I). The particle spectrum was generated from the $\Delta E$ and $E$ pulse information by the technique discussed in Appendix A.

Fig. 13. Particle spectrum of fragments ejected at $20^{\circ}$ to beam in bombardment of $\mathrm{U}$ target with $5.0 \mathrm{GeV}$ protons. Fragments penetrating Al absorber $\left(690 \mathrm{mg} / \mathrm{cm}^{2}\right.$ ) were measured with 3-element telescope (see Table I). The particle spectrum was generated from the $\Delta \mathrm{E}$ and $\mathrm{E}$ pulse information by the technique discussed in Appendix A.

Fig. 14. High-energy part of energy spectrum of ${ }^{6} \mathrm{Li}$ and ${ }^{7} \mathrm{Li}$ fragments ejected at $20^{\circ}$ to the proton beam. The 4 sets of points refer to measurements with different aluminum absorbers in front of first $\Delta \mathrm{E}$ detector $(35.7,432$, 690 , and $1295 \mathrm{mg} / \mathrm{cm}^{2}$, respectively). The $U$ target data points for the $35.7 \mathrm{mg}$ absorber are plotted $20 \%$ low. The solid curves were sketched in to represent the smoothed trend of the data. The dashed lines represent the same MAXVAP curves calculated for $\mathrm{Ag}$ as the one for $20^{\circ}$ shown in Fig. 3, adjusted for ${ }^{6}{ }_{\mathrm{Li}}$ and ${ }^{7} \mathrm{Li}$ isotopic composition. 
Fig. 15. High energy part of energy spectrum for ${ }^{7}$ Be fragments ejected at $20^{\circ}$ to the proton beam. Lowest energy set of Ag points are reproduced from Fig. 4. The other sets of points came from measurements with 4 different aluminum absorbers in front of detector telescope. Solid curves represent smoothed trend of data. Dashed line is the MAXVAP curve calculated for $\mathrm{Ag}$ for $20^{\circ}$ reproduced from Fig. 4. The $80-140 \mathrm{MeV}$ points for $\mathrm{U}$ are plotted $20 \%$ low.

Fig. 16. Energy spectrum of ${ }^{9,10}$ Be fragments ejected at $20^{\circ}$ to the proton beam. Lower energy set of points reproduced from Fig. 5 (Ag target). Higher energy sets of data taken with 3-element telescopes with $35.7 \mathrm{mg} / \mathrm{cm}^{2} \mathrm{Al}$ absorber in front. U data plotted 20\% low. Dashed curve represents calculation based on evaporation oode MAXVAP. This curve is the same as shown in Fig. 5 for $20^{\circ}$.

Fig. 17. Energy spectrum of B fragments ejected at $20^{\circ}$ to the proton beam. See caption of Fig. 16 for further details.

Fig. 18. Energy spectrum of $\mathrm{C}$ fragments ejected at $20^{\circ}$ to the proton beam. See caption of Fig. 16 for further details.

Fig. 19. Energy distributions at $20^{\circ}$ to the beam for ${ }^{3} \mathrm{He}$, ${ }^{4} \mathrm{He}$, and ${ }^{6} \mathrm{He}$ ejected from a Ag target. Solid curves represents data from Ref. 2. Squares and triangles show new data.

Fig. 20. Topographical display of particle total energy $E_{T}$ versus $\Delta E$ for particles emitted at $90^{\circ}$ to beam from Ag target. $\Delta \mathrm{E}$ data taken with $16 \mu \mathrm{m}$ silicon detector. Display based on $128 \times 128$ grid, whereas $E_{T}$ versus $\Delta E$ data used in final analysis were stored in a $1024 \times 1024$ matrix. The data can be represented by an equation of the form $E_{T}=k \times \exp (-m \triangle E)$. 
Fig. 2l. Particle spectra generated by the PICAL program using algorithm (A-5). In the upper curves the exponent $b$ was set at $1.0,1.5$, and 2.0 , respectively. In the bottom curve the above range of $b$ values were used in the particle section in which they produced the best resolution.

Fig. 22. Topographical display of $E_{T}$ versus PI. Data are the same as those in Fig. 20. PI was calculated with algorithm (A-5) with the same b parameter range as given in Fig. 21 . 


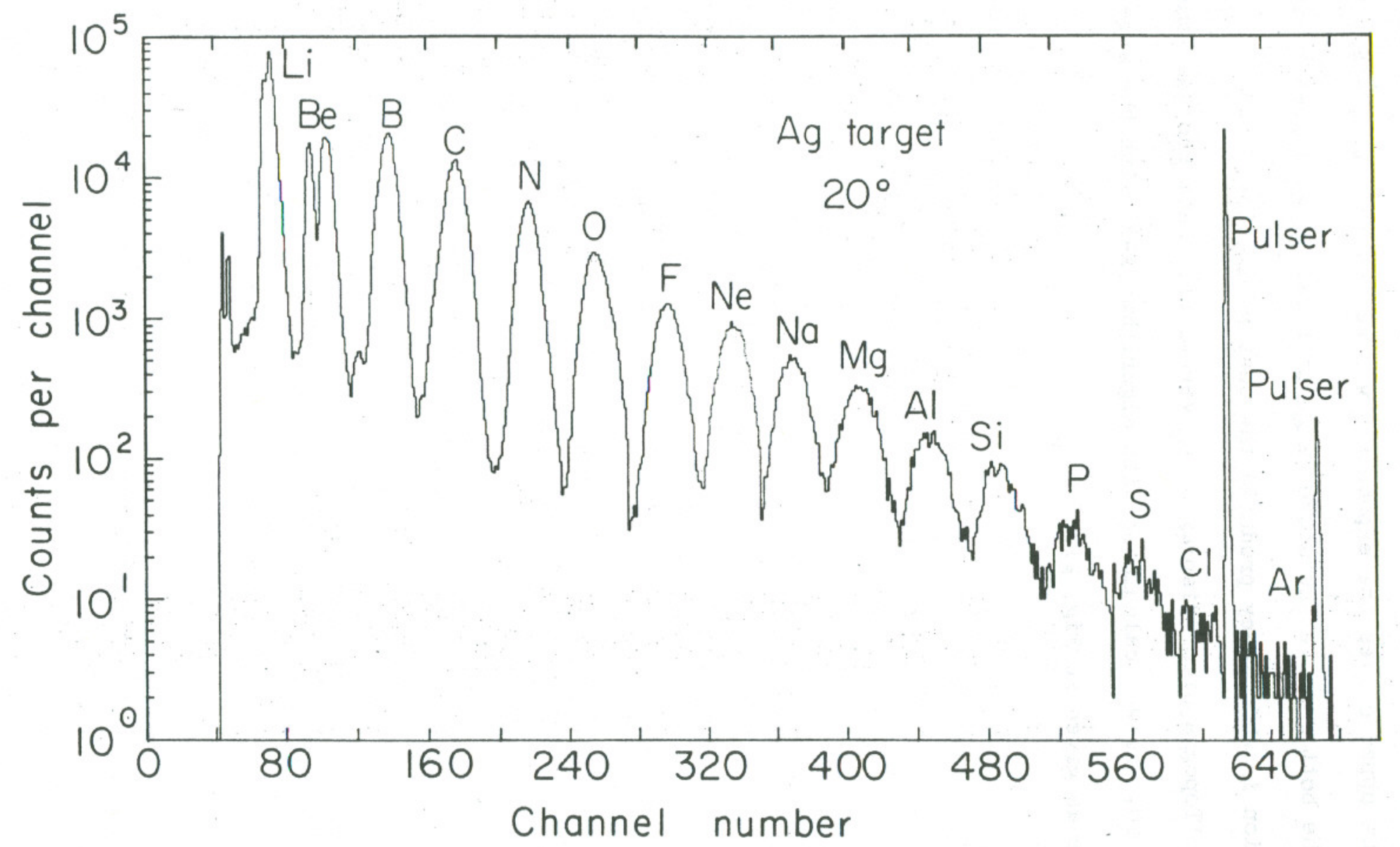

Fig. 1 


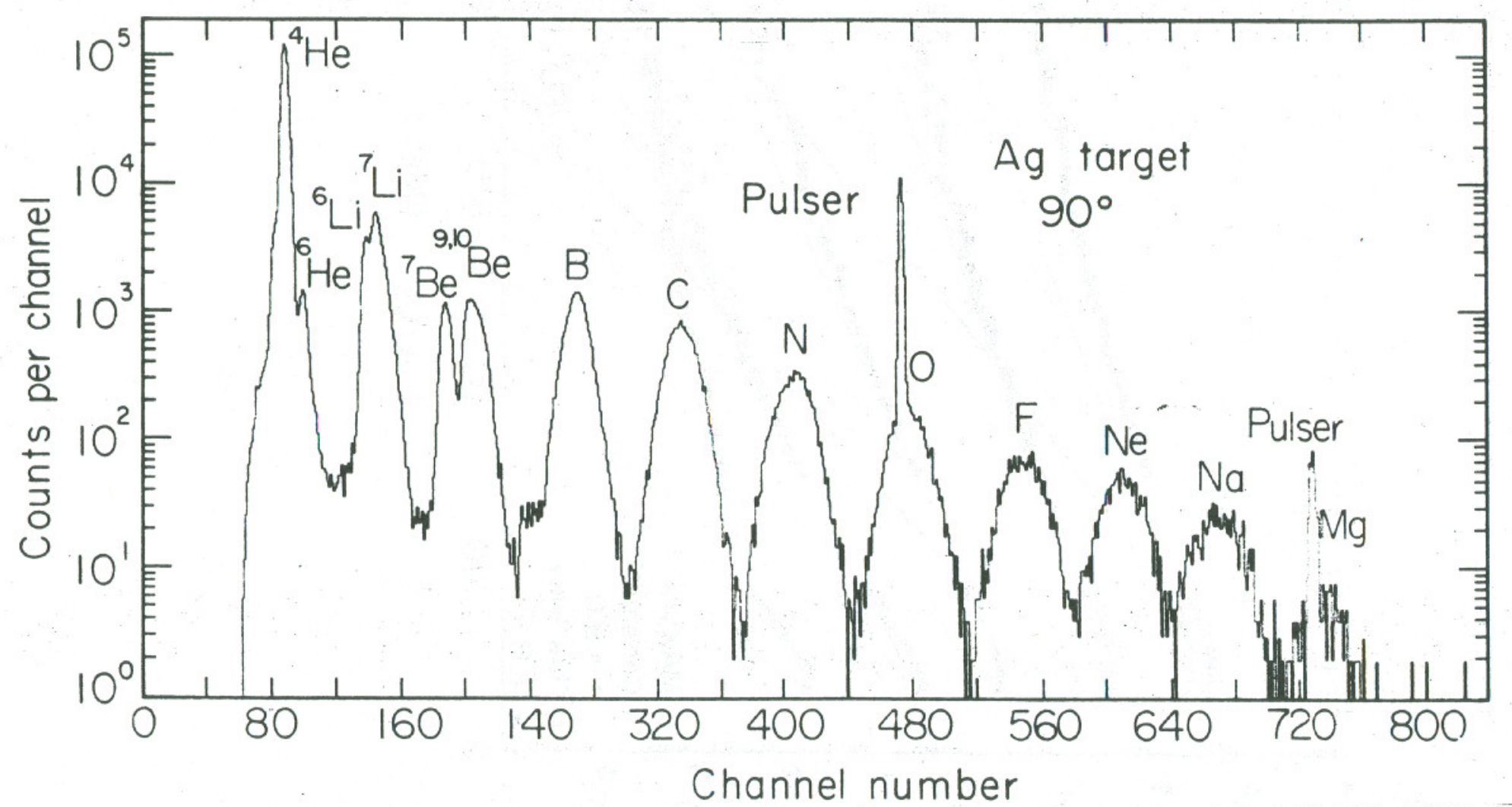

Fig. 2 


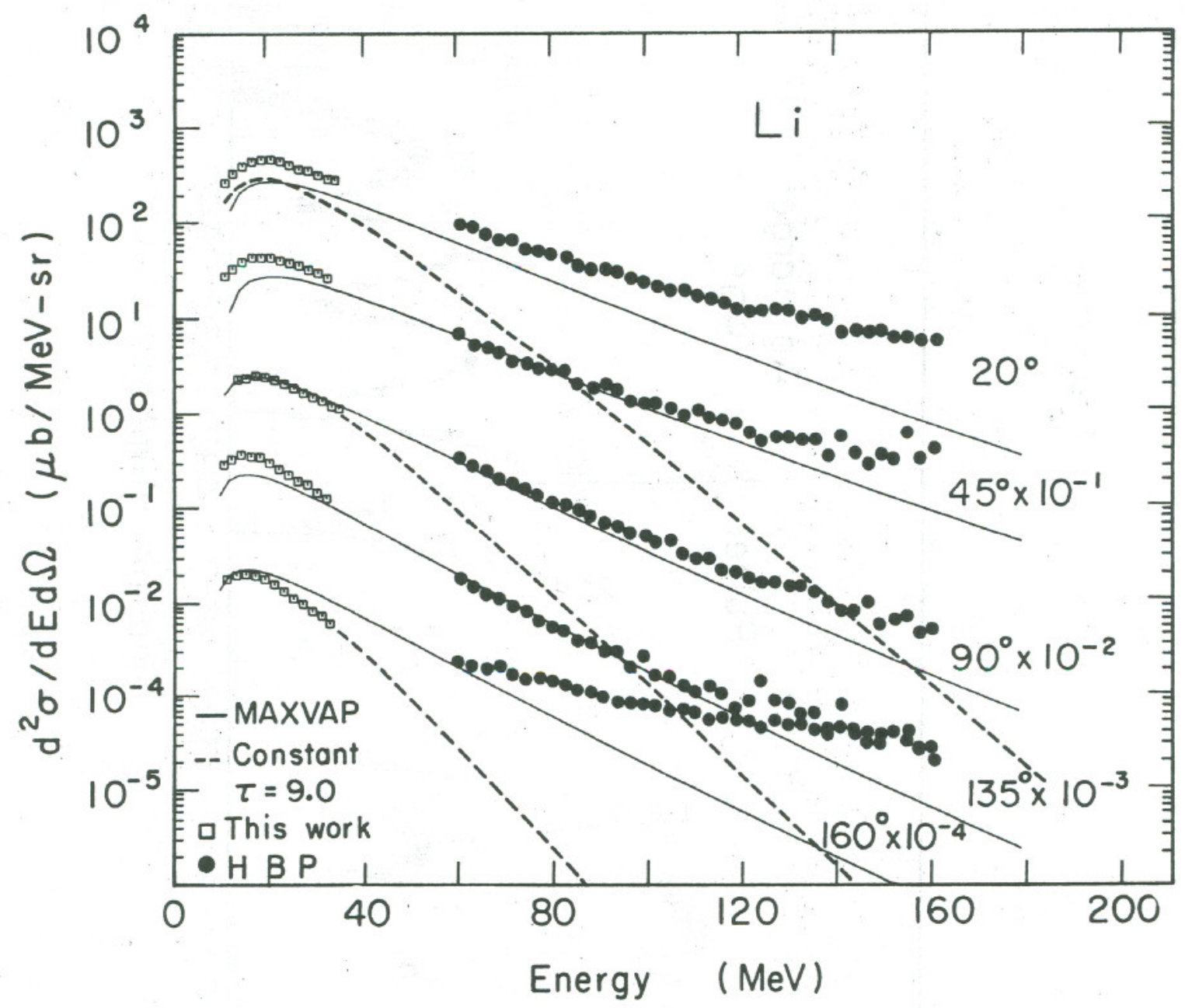

XBL728-3833

Fig. 3 


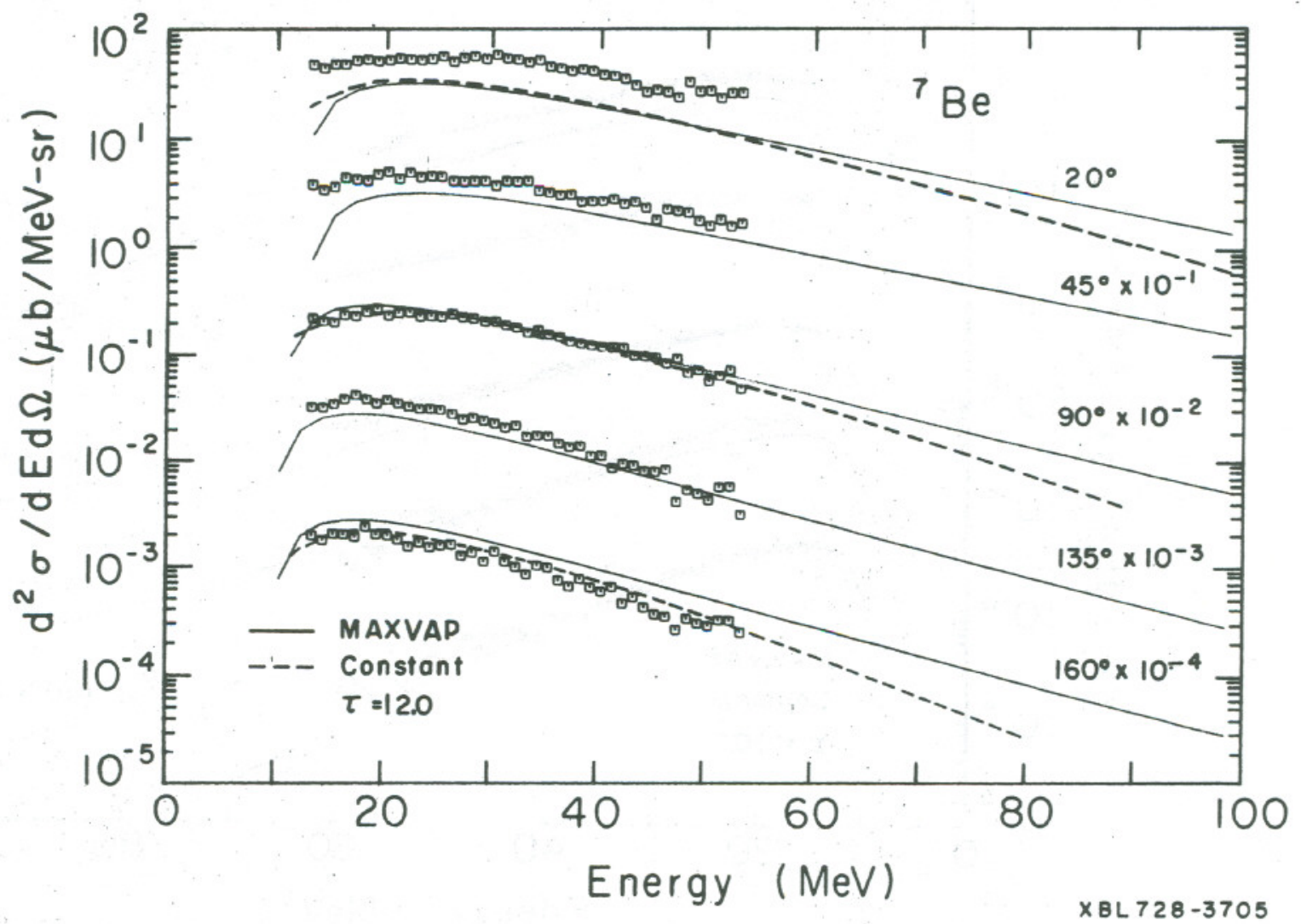

Fig. 4 


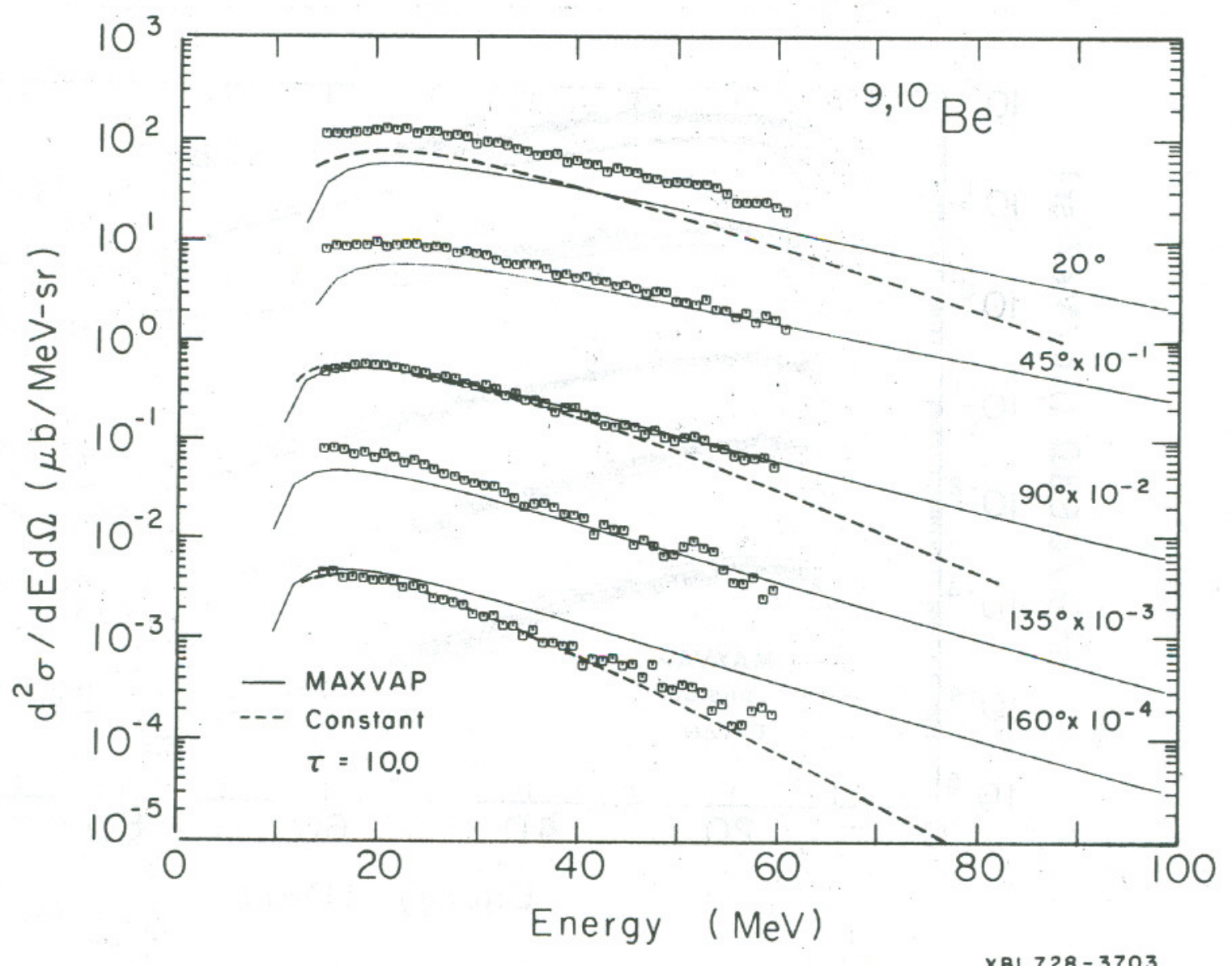

Fig. 5 


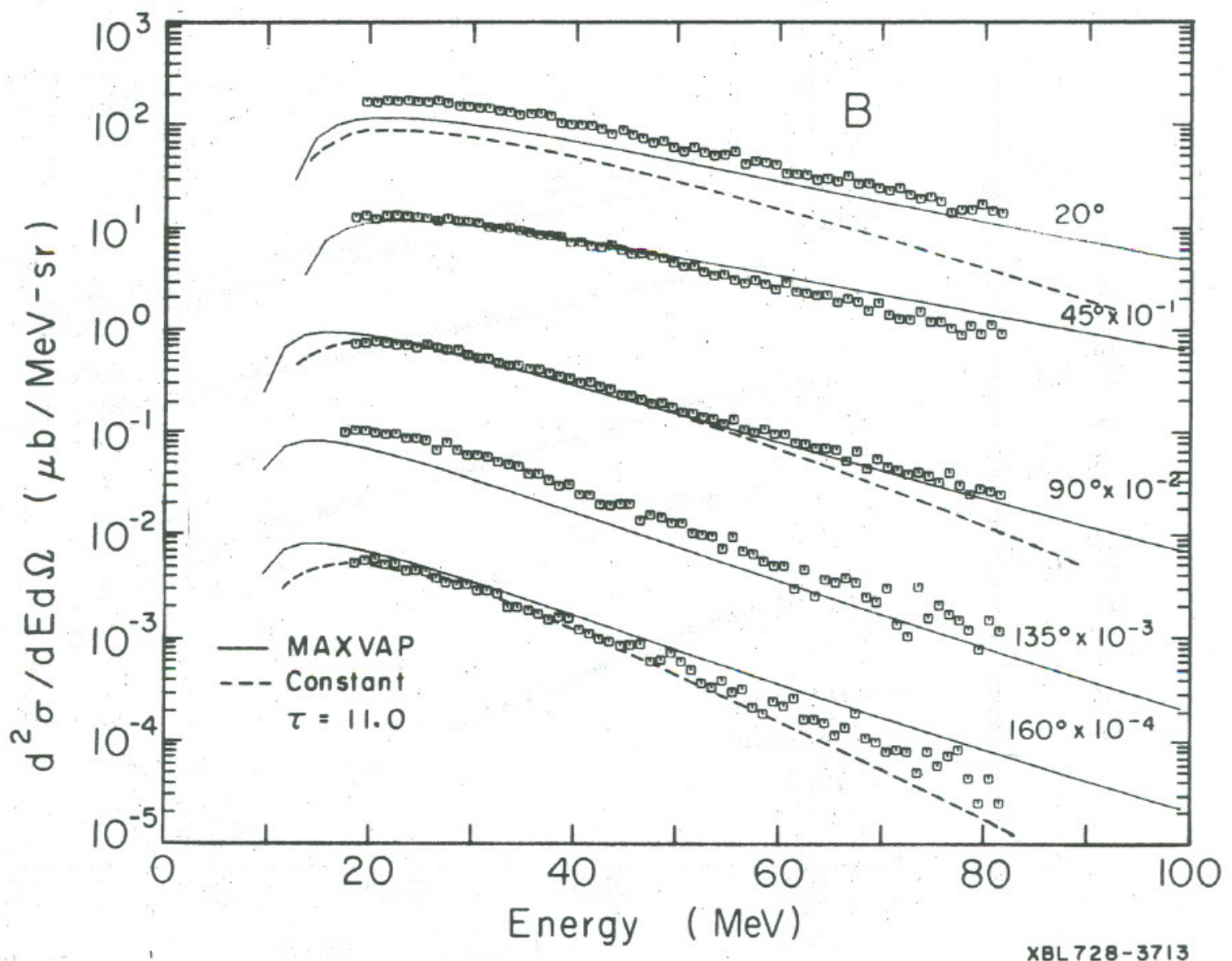

Fig. 6 


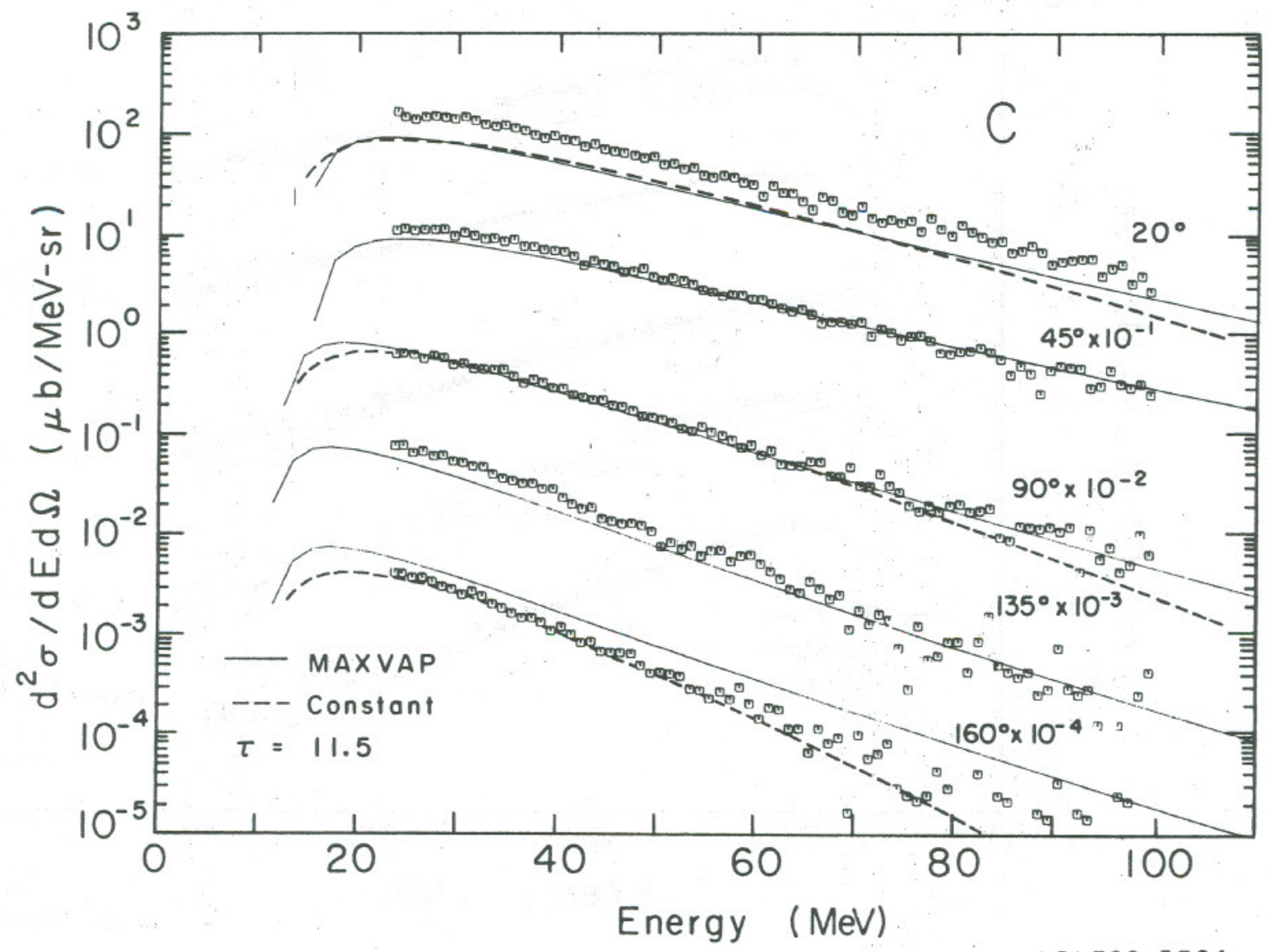

Fig. 7 


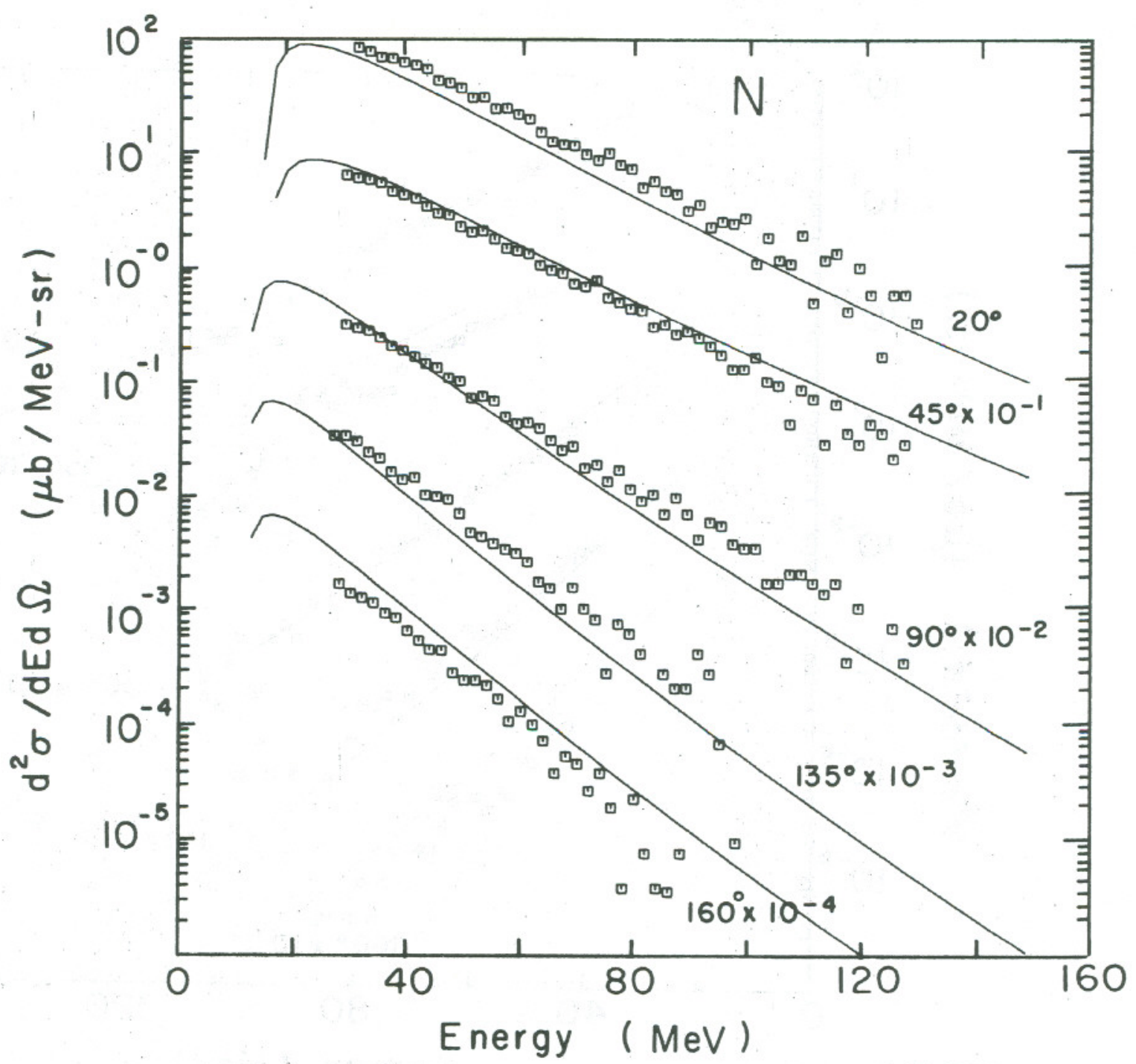

XBL 728-3696

Fig. 8 


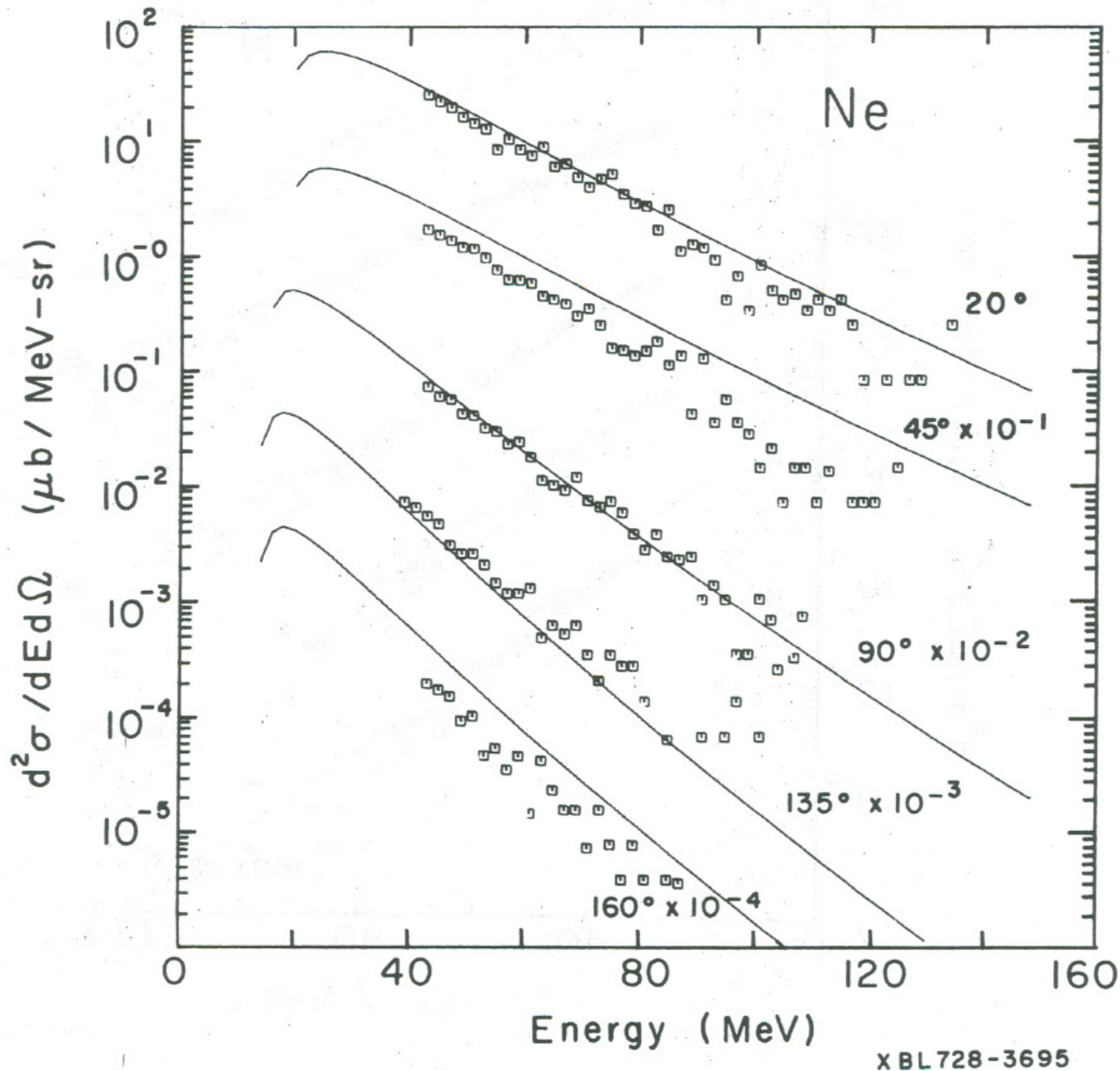

Fig. 9 


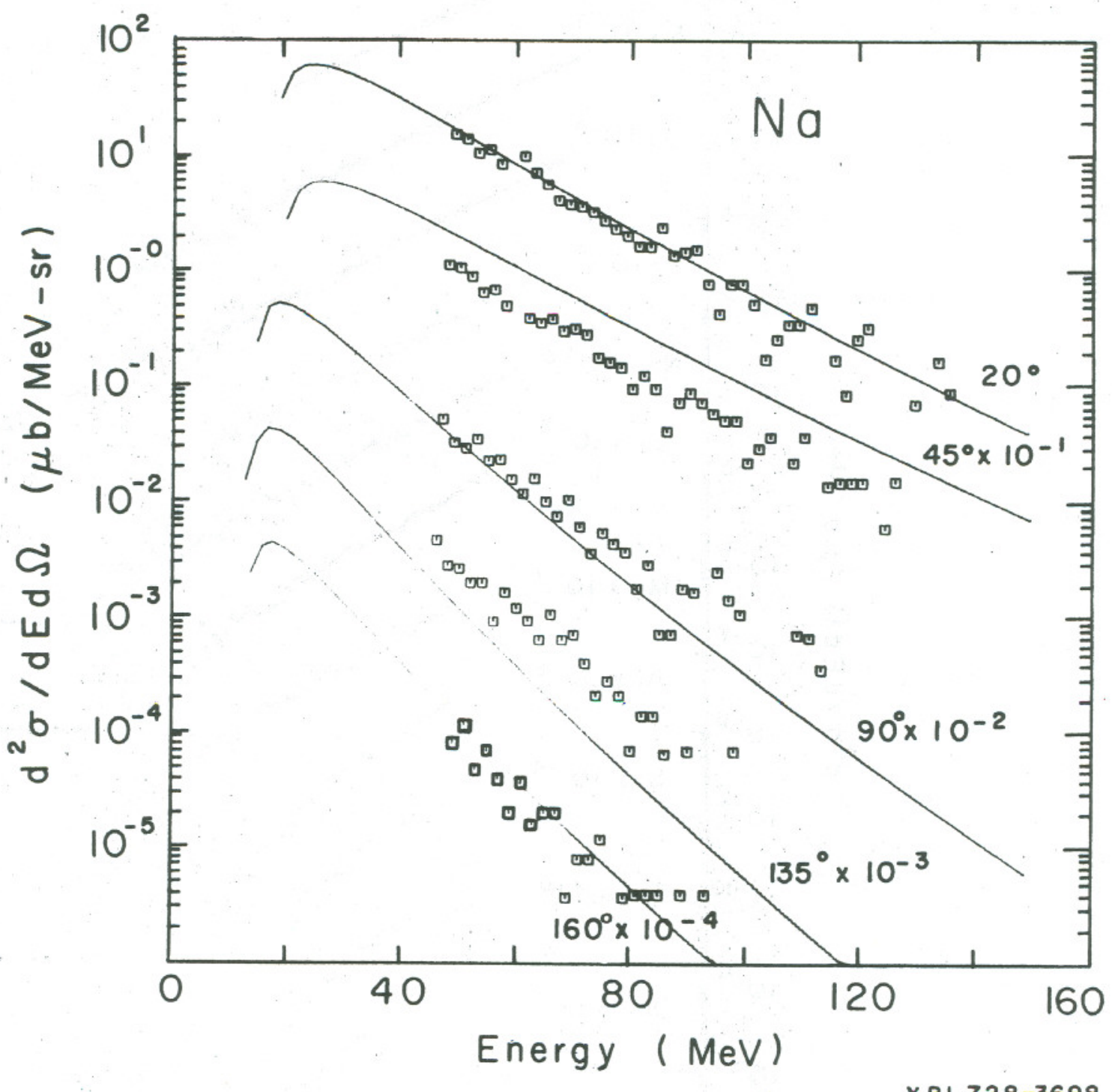

Fig. 10 


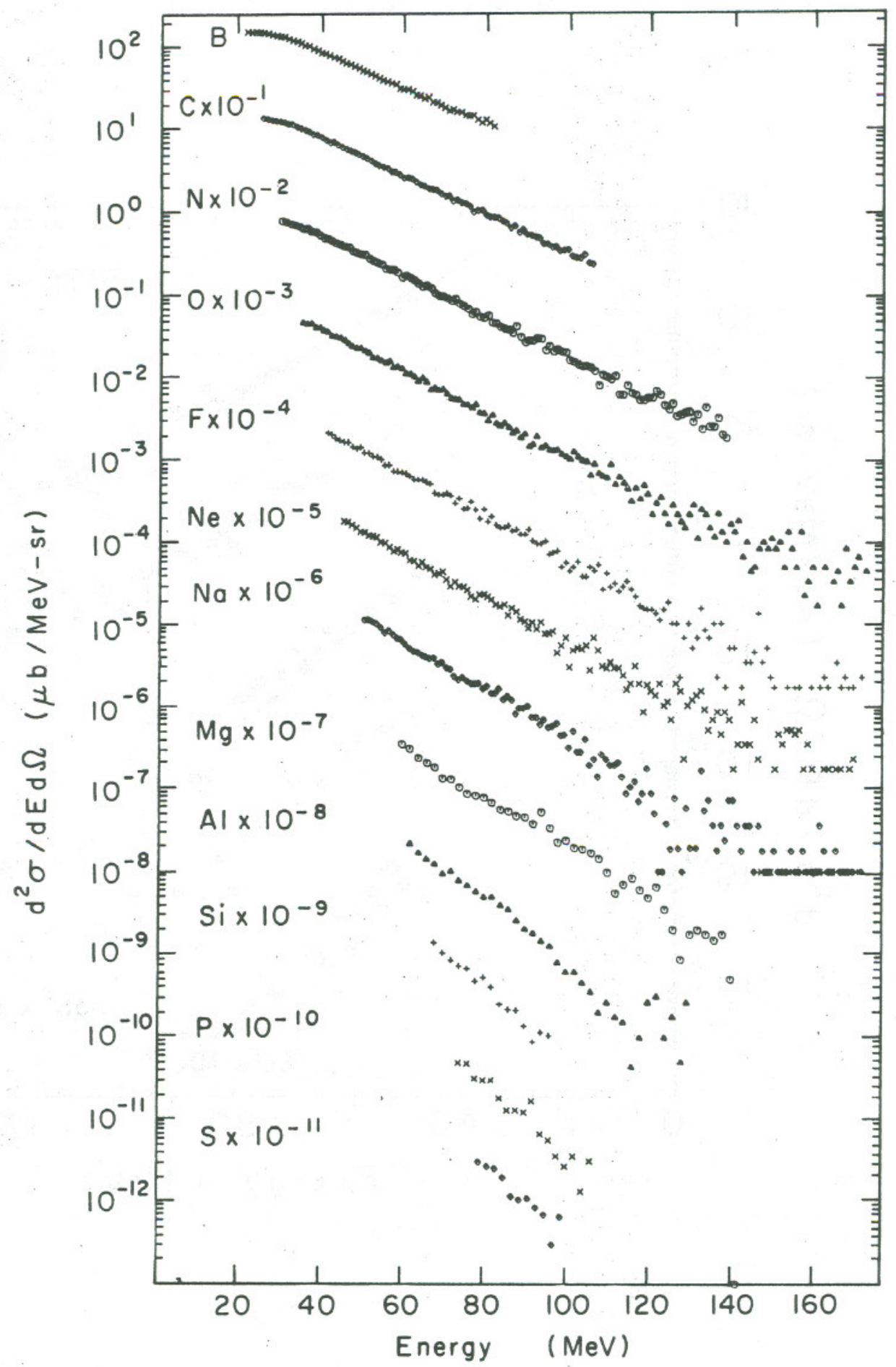

XBL729-4039

Fig. 11 


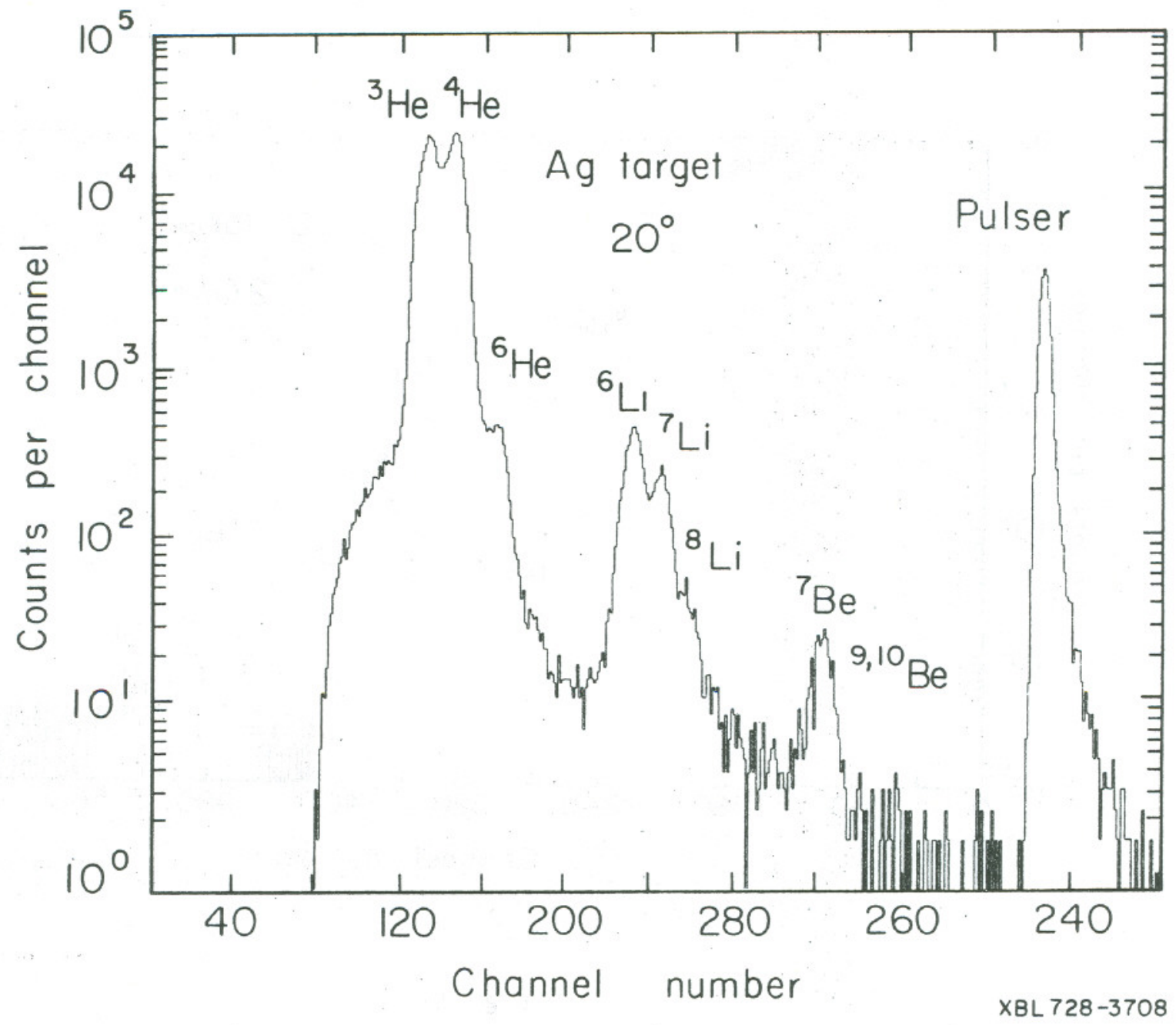

Fig. 12 


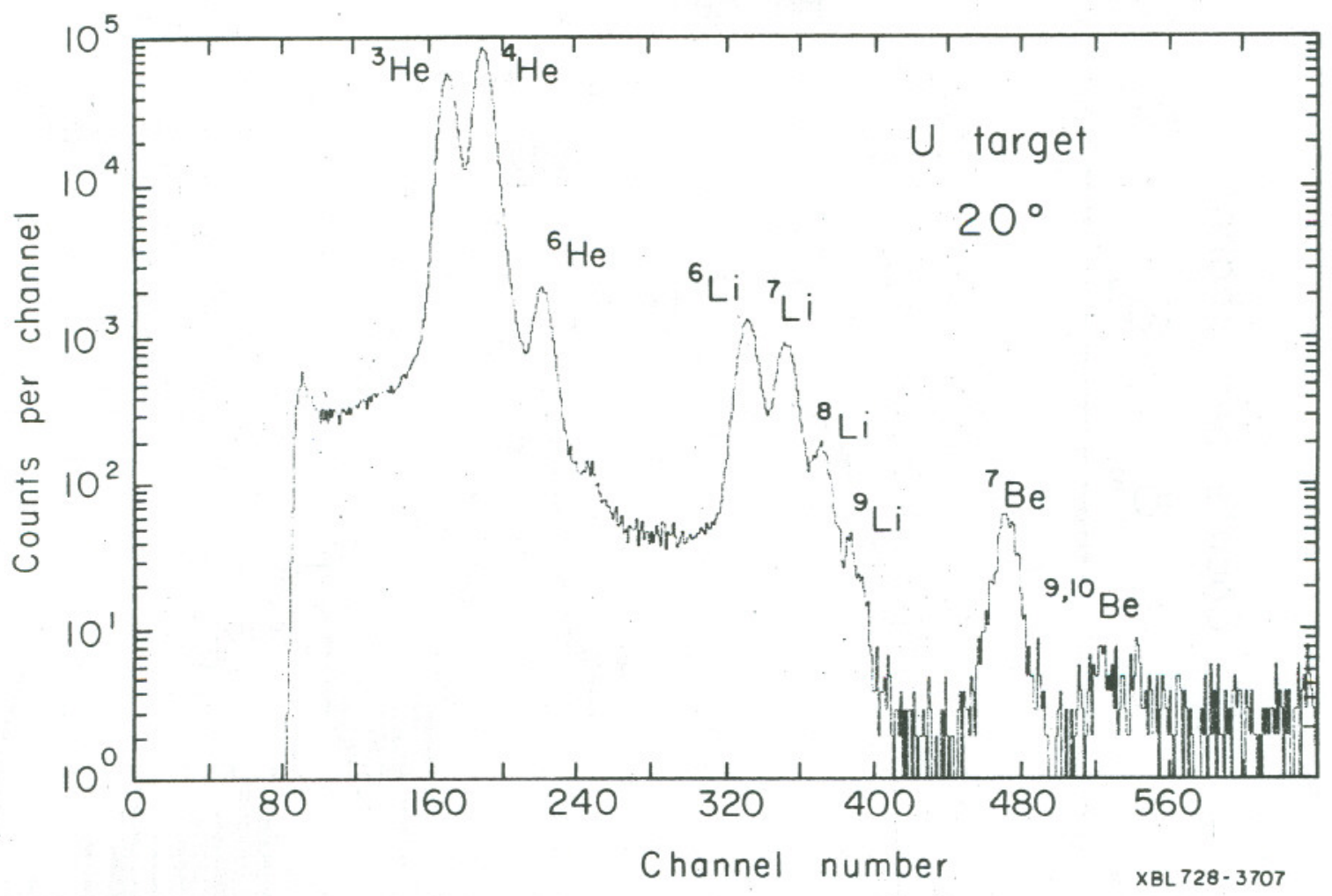

Fig. 13 


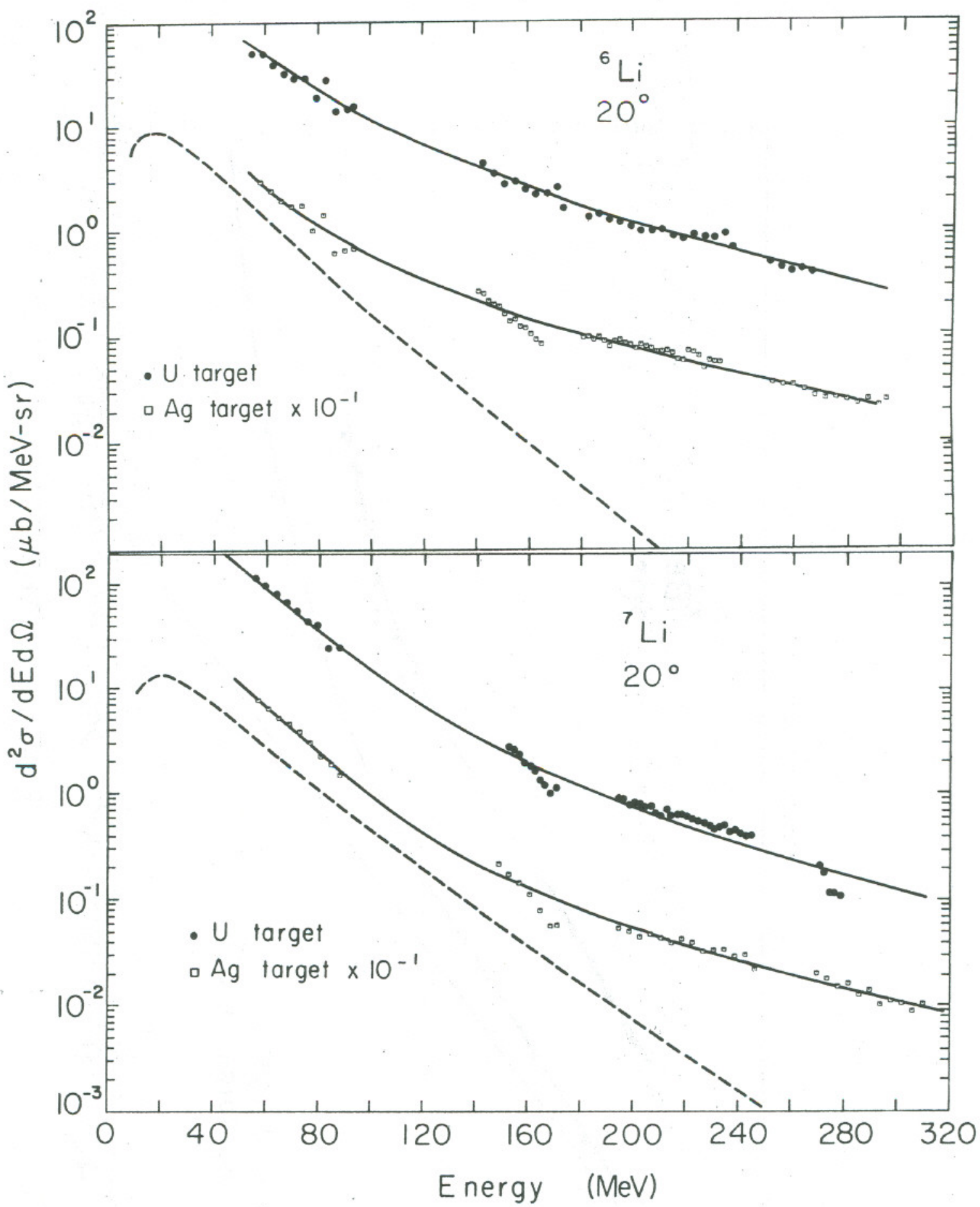

XBL $728-3840$

Fig. 14 


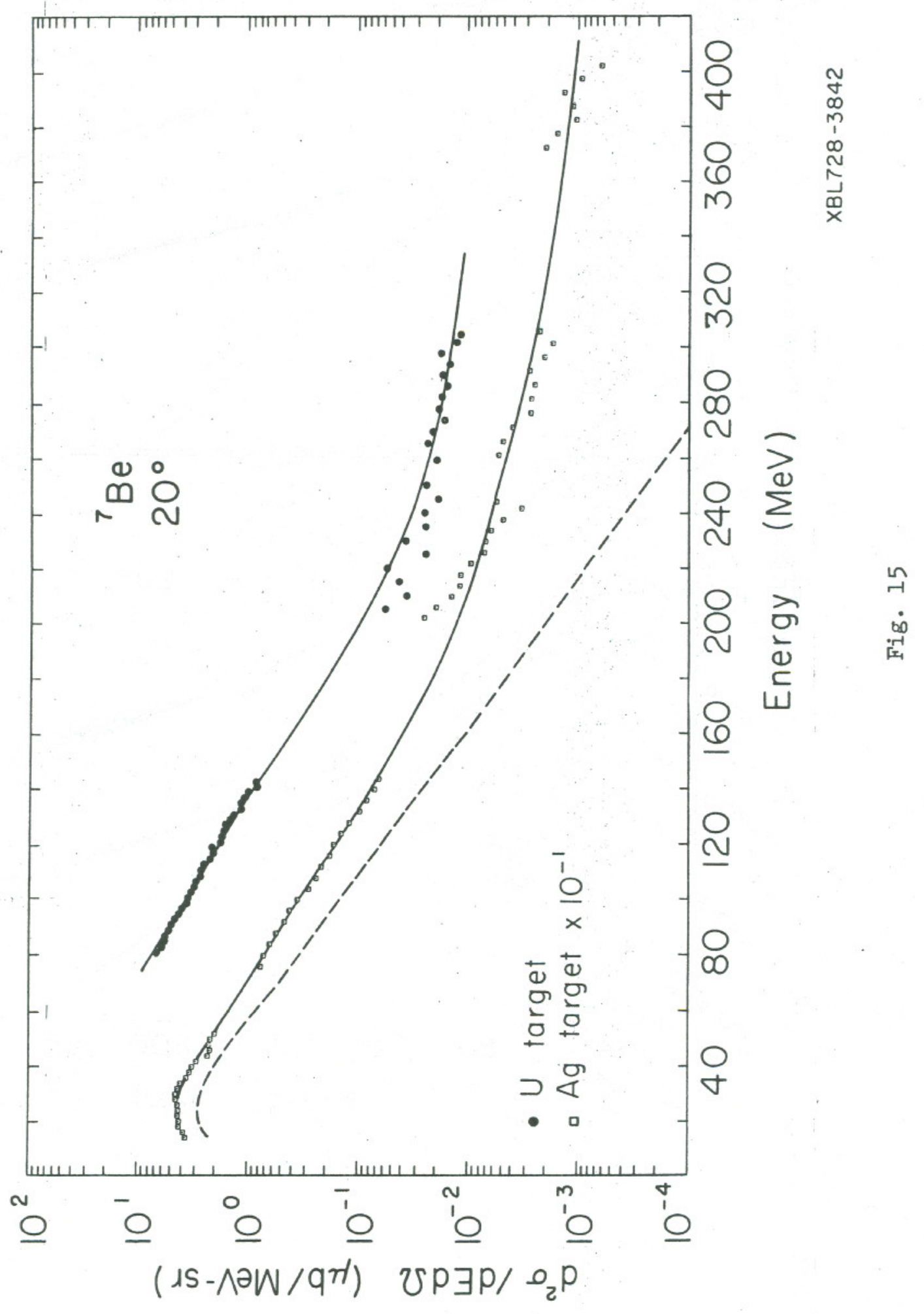




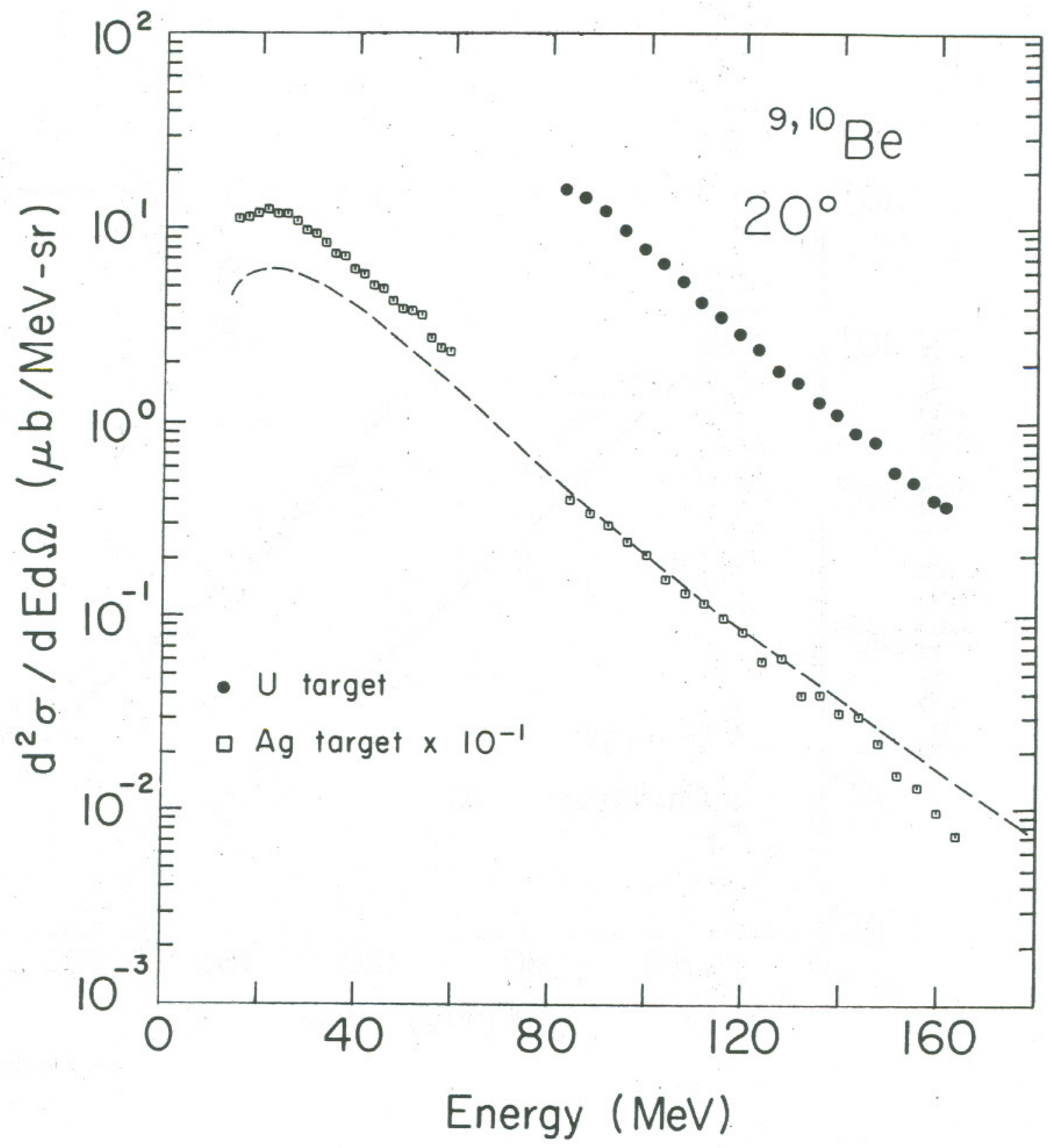

XBL $728-3837$

Fig. 16 


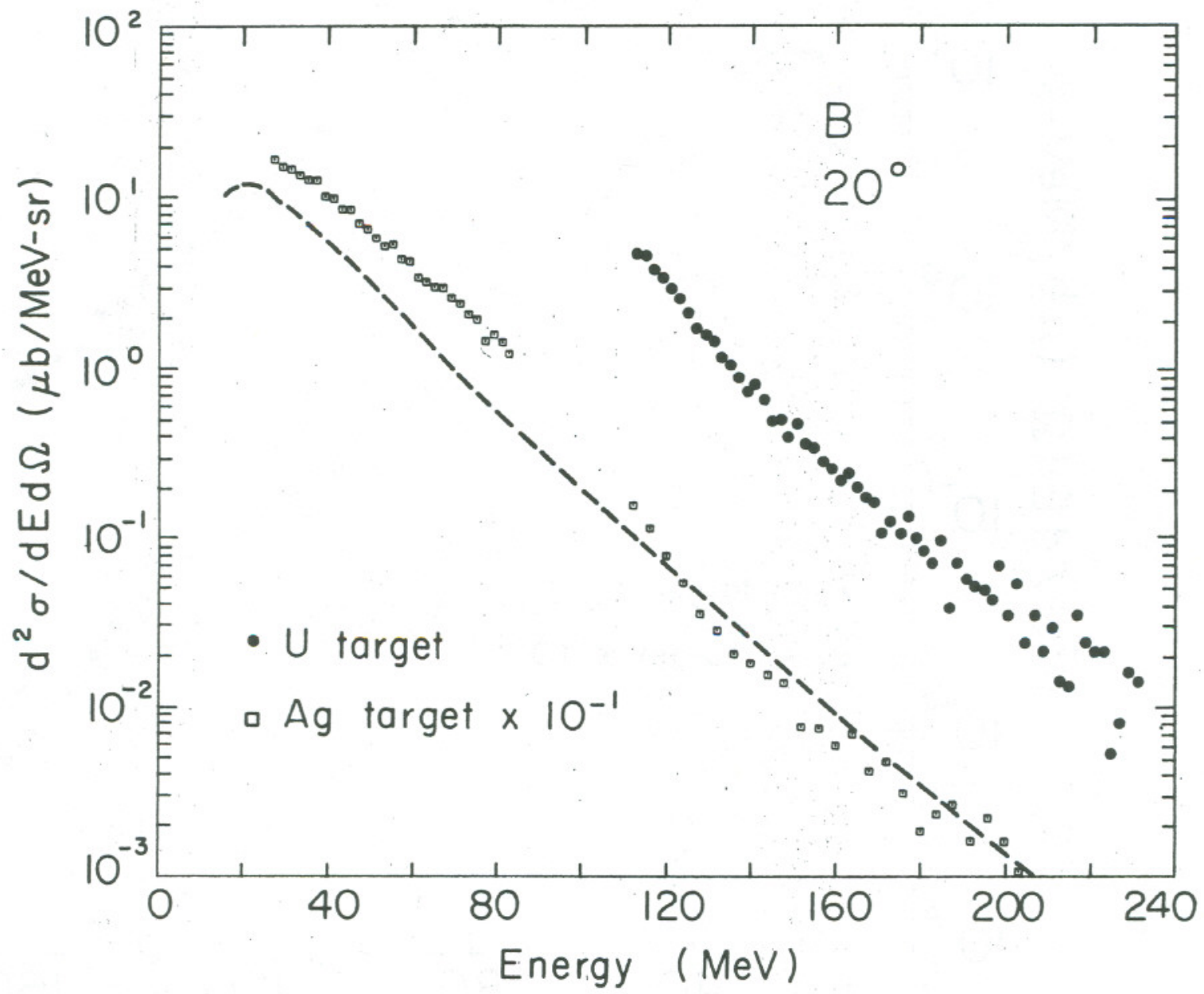

XBL 728-3834

Fig. 17 


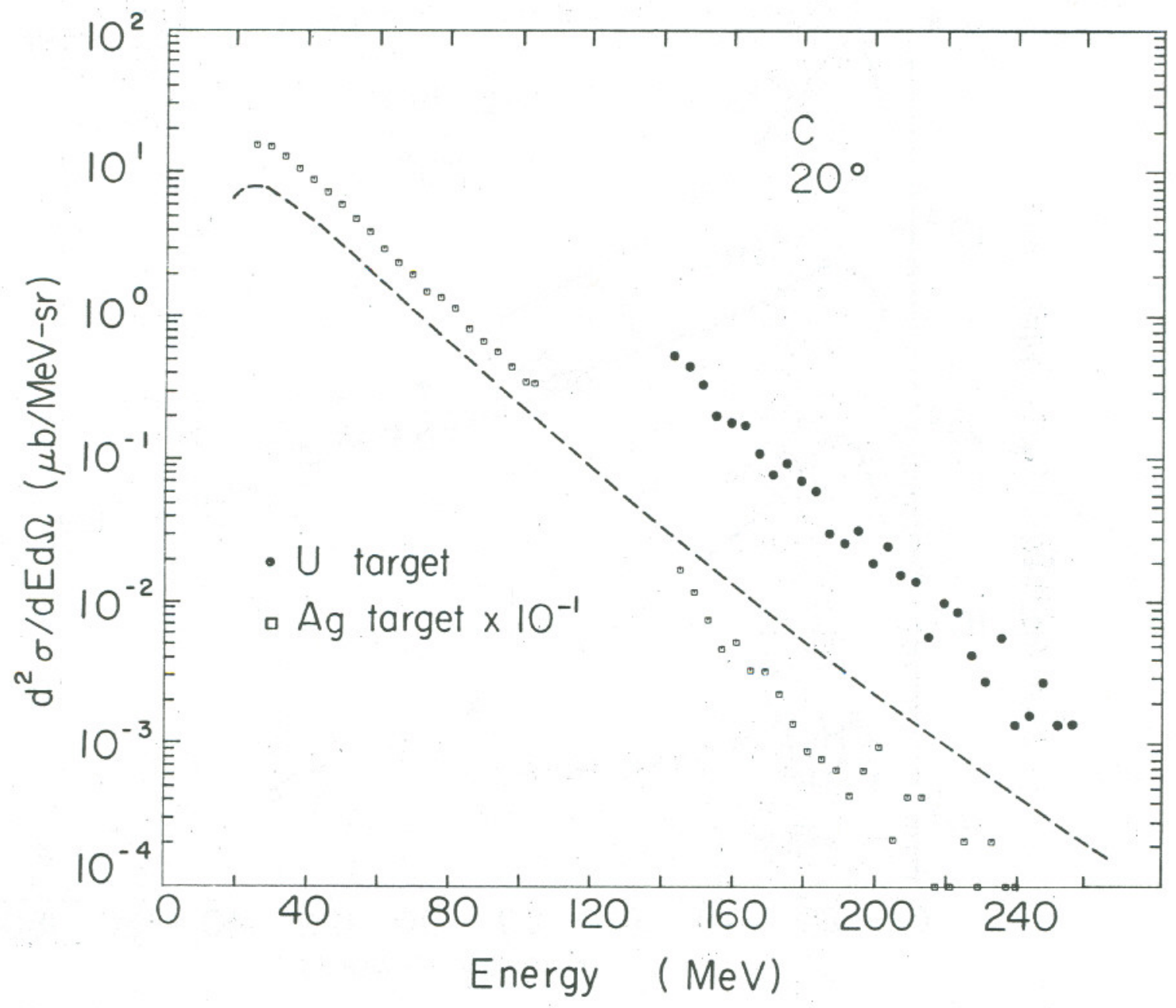

XBL 728-3835

Fig. 18 


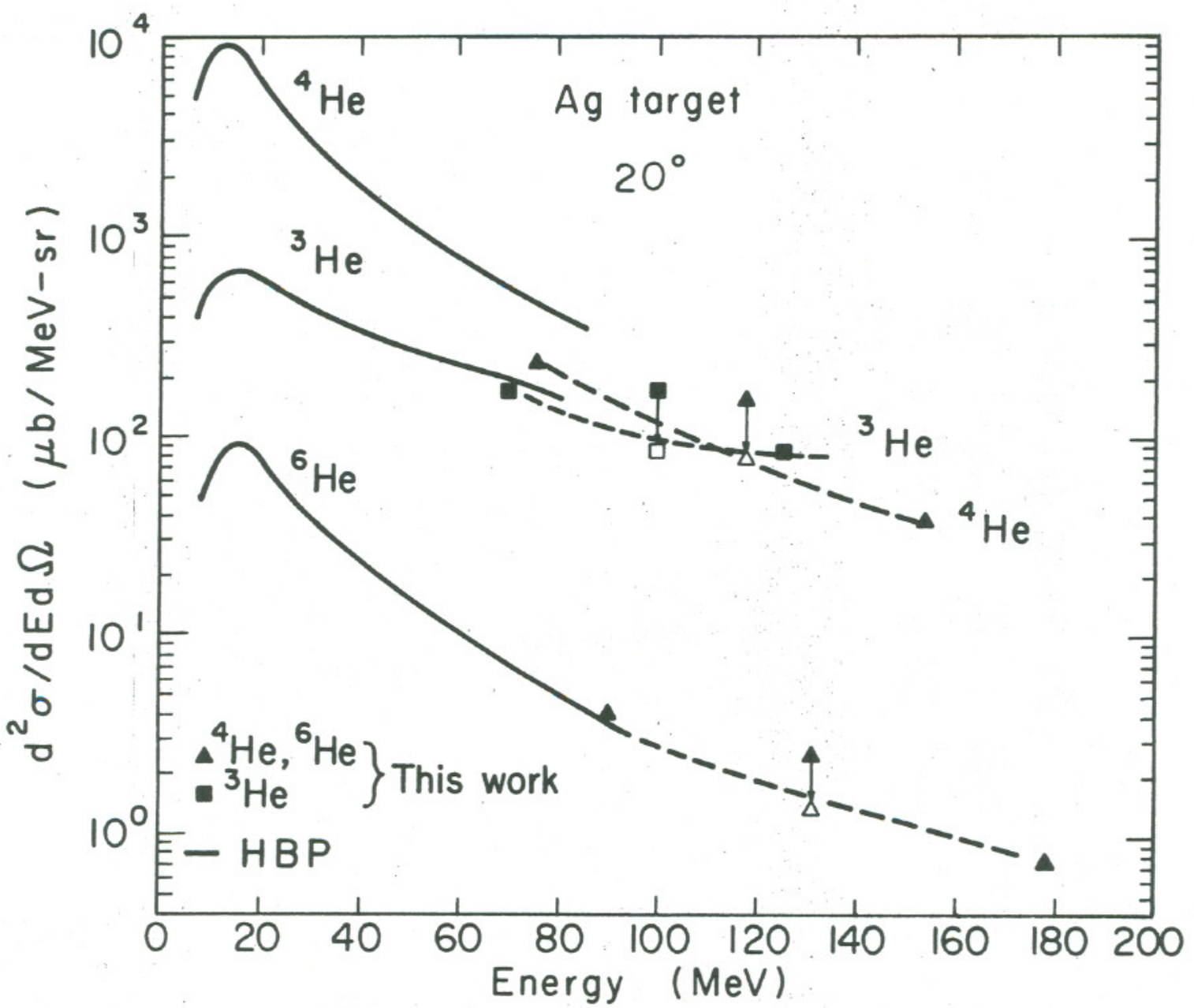

XBL729-4040

Fig. 19 
I BI -663

$-59-$

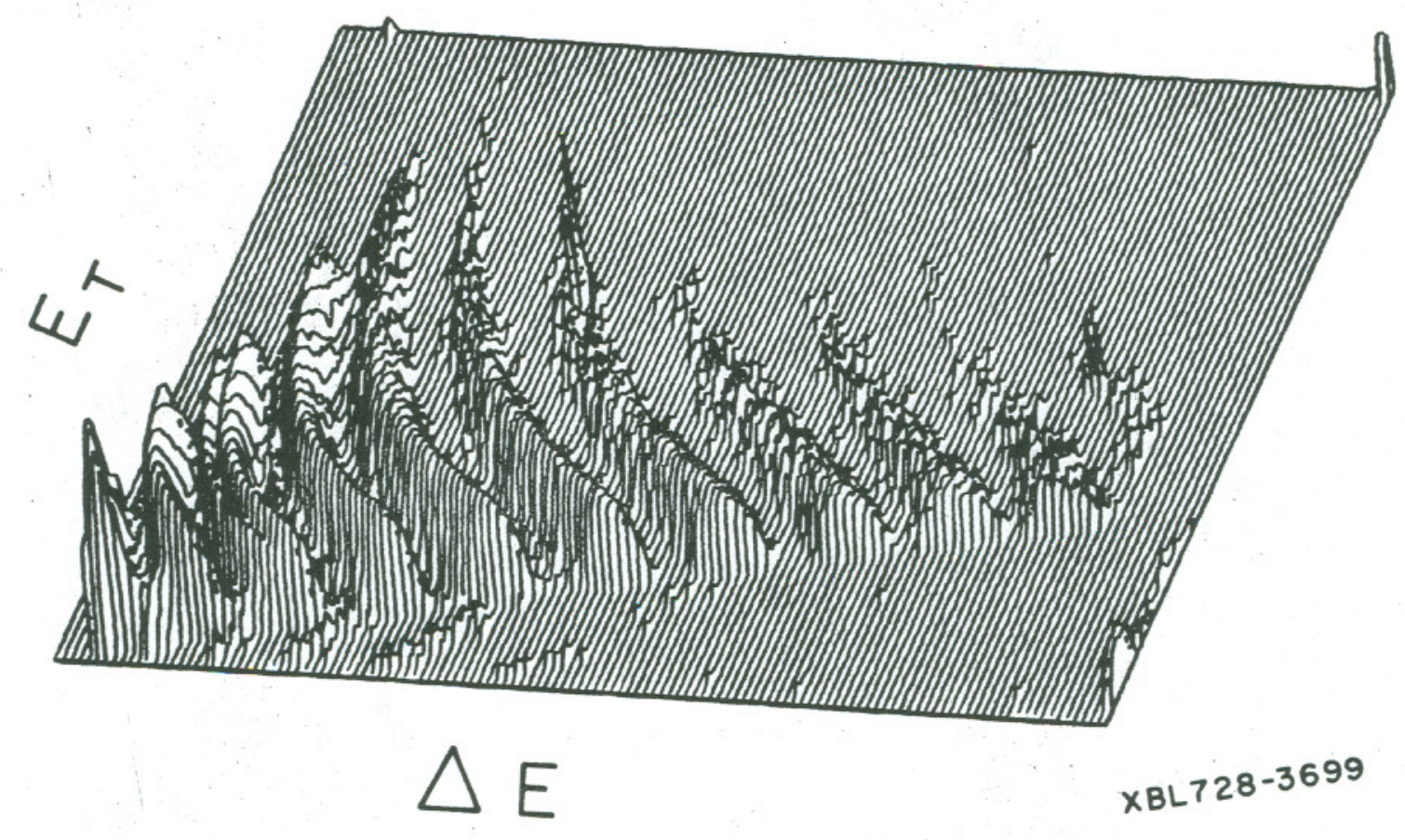

Fig. 20 


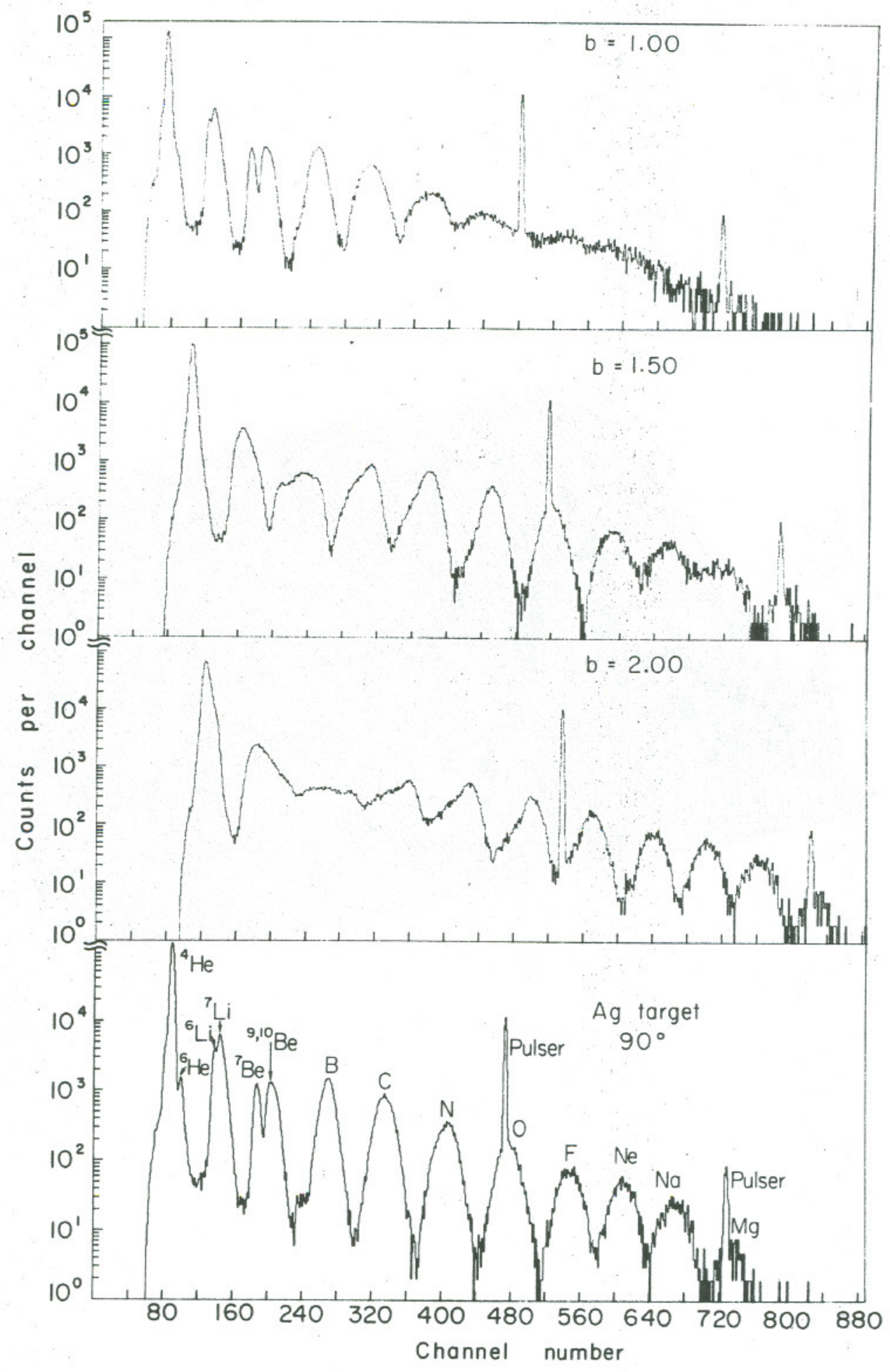

$\times B L 728-3841$

Fig. 21 


\section{LEGAL NOTICE}

This report was prepared as an account of work sponsored by the United States Government. Neither the United States nor the United States Atomic Energy Commission, nor any of their employees, nor any of their contractors, subcontractors, or their employees, makes any warranty, express or implied, or assumes any legal liability or responsibility for the accuracy, completeness or usefulness of any information, apparatus, product or process disclosed, or represents that its use would not infringe privately owned rights. 